\title{
Effective permittivity of dense random particulate plasmonic composites
}

\author{
Satvik N. Wani, ${ }^{1}$ Ashok S. Sangani, ${ }^{1,2}$ and Radhakrishna Sureshkumar ${ }^{1,3, *}$ \\ ${ }^{1}$ Department of Biomedical and Chemical Engineering, Syracuse University, Syracuse, New York 13244, USA \\ ${ }^{2}$ National Science Foundation, Arlington, Virginia 22230, USA (On leave from Syracuse University) \\ ${ }^{3}$ Department of Physics, Syracuse University, Syracuse, New York 13244, USA \\ ${ }^{*}$ Corresponding author: rsureshk@syr.edu
}

Received February 14, 2012; accepted March 24, 2012;

posted April 13, 2012 (Doc. ID 163084); published May 30, 2012

\begin{abstract}
An effective-medium theory (EMT) is developed to predict the effective permittivity $\varepsilon_{\text {eff }}$ of dense random dispersions of high optical-conductivity metals such as $\mathrm{Ag}, \mathrm{Au}$, and $\mathrm{Cu}$. Dependence of $\varepsilon_{\text {eff }}$ on the volume fraction $\phi$, a microstructure parameter $\kappa$ related to the static structure factor and particle radius $a$, is studied. In the electrostatic limit, the upper and lower bounds of $\kappa$ correspond to Maxwell-Garnett and Bruggeman EMTs, respectively. Finite size effects are significant when $\left|\beta^{2}(k a / n)^{3}\right|$ becomes $O(1)$, where $\beta, k$, and $n$ denote the nanoparticle polarizability, wavenumber, and matrix refractive index, respectively. The coupling between the particle and effective medium results in a red-shift in the resonance peak, a nonlinear dependence of $\varepsilon_{\text {eff }}$ on $\phi$, and Fano resonance in $\varepsilon_{\text {eff }}$. (c) 2012 Optical Society of America

OCIS codes: $\quad 160.4236,160.4670,260.2065,260.2110,260.3910,260.5740$.
\end{abstract}

\section{INTRODUCTION}

Nanoparticulate plasmonic composite materials have recently become technologically important, especially in the growing interdisciplinary fields of plasmonics and metamaterials [므므. Fabrication of such plasmonic nanocomposites is accomplished through well-established methods such as surfactant mediated self-assembly [7], laser dewetting of thin films [8], sol-gel assembly [9-11], ion-implantation [12,13], and vacuum evaporation of thin films [14], among others. Technologically, such composites are important in the fields of energy harvest-

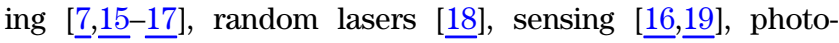
catalysis $[20]$, etc. Free electrons in noble metal nanoparticles (NPs) give rise to a characteristic plasmon resonance wherein the NPs absorb and scatter radiation with a marked intensity [3,21-23]. The linear optical response of such materials can be described by Maxwell equations in both the dispersed and continuous phases when the particle size is greater than a few nanometers. In the case of metal NPs in a transparent medium, the matrix phase can be treated as a dielectric with real electrical permittivity $\varepsilon_{m}$, while the NPs should be treated as materials with complex, frequency-dependent permittivity $\varepsilon_{p}$. A plasmon resonance occurs for $\mathfrak{R}\left(\varepsilon_{p} / \varepsilon_{m}\right)=-2$ in the case of spheres. The overall optical response of such composite materials can be determined by numerically solving Maxwell equations in both phases subject to the continuity of tangential components of magnetic and electric fields at the interface of the embedded particles and the surrounding matrix phase. Such calculations are possible by utilizing computational techniques such as the finite difference time domain method [24-26]. However, substantial computational effort is required, especially near resonant frequencies where steep field gradients necessitate the use of very fine spatial resolution [26]. Hence, theories capable of accurately predicting the average optical properties of random composites could be a valuable tool in the knowledge-based design of plasmonic composites.

Composite media are inherently inhomogeneous. Hence, their average electromagnetic behavior depends on the permittivities and volume fractions of the constituent components. Effective-medium theories have been used to parameterize the properties of such media [27]. In the case of monodisperse spherical particulate composites, where the particle radius $a$ is much smaller than the wavelength of exciting radiation $\lambda$, the effective permittivity can be modeled under the quasistatic approximation wherein the wave nature of the electromagnetic fields can be neglected. Specifically, for the case of plasmonic composites in the optical frequency range, the magnetic response in the optical range is the same as that of vacuum, and the classical Maxwell-Garnett theory (MGT) [28] can be used to predict the effective permittivity. Similarly, in the case $\lambda \ll a$, ray optics can be utilized. However, for nanoscopic plasmonic composites in the optical range, $\lambda$ can be $O(a)$ or $k a \equiv 2 \pi \sqrt{\varepsilon} a / \lambda$ is $O(1)$. Hence, the effects of diffraction and scattering by the NPs become significant and simple models designed for either one of the extreme cases are not applicable. Extended Maxwell-Garnett theories have been developed in the literature for this regime [29-32]. However, they are correct only up to $O(\phi)$ ( $\phi$ : volume fraction of the dispersed phase) and do not account for the effect of microstructure on the permittivity. In this work, we have developed a self-consistent theoretical framework for the prediction of the effective linear optical properties of dense random monodisperse spherical particulate plasmonic composites with particle size on the order of the exciting wavelength of radiation. This effective-medium theory (EMT) is a method employed for the accurate prediction of sound attenuation and phase speed in acoustically resonant monodisperse suspensions of microspheres by Spelt et al. [33]. The microstructure information is incorporated through the static 
structure factor $S(\mathbf{0})$. It has been shown that the leading order correction term in terms of the particle volume fraction to the velocity field in Stokes flow, i.e., "slow" flow of a viscous liquid in which inertial forces are negligibly small, for infinite randomly distributed monodisperse spheres depends linearly on the static structure factor $S(\mathbf{0})$ [34]. Both Stokes flow equations and Maxwell wave equations take the form of a vector Helmholtz equation for the fluid velocity and electric field, respectively. Hence, the methodology developed by Spelt et al. [33] can be adapted to derive an EMT for electromagnetic wave propagation problems in heterogeneous media. Specifically, the composite medium is represented by a layered structure in which the particle, in its immediate vicinity, is surrounded by the dielectric matrix up to a distance $R$, which is a function of $S(\mathbf{0})$. The structure is assumed to be embedded in an effective continuum whose permittivity $\varepsilon_{\text {eff }}$ is determined by the self-consistent solution of Maxwell equations. The EMT is mathematically identical to the Maxwell-Garnett model in the limit as the particle diameter $d_{p}$ and volume fraction $\phi$ approach zero. However, for finitely large volume fractions, the variations in the permittivity with respect to $\phi$ and $d_{p}$ are highly nonlinear.

The paper is organized as follows. Problem formulation and the derivation of the ensemble-averaged Maxwell equations are presented in Section 2. The EMT is discussed in Subsection 2.A. Section 3 contains a summary of the solution technique and computational methods used to calculate the conditionally averaged electric field. Analytical and numerical results are discussed in Section 4 . Results of the scalar EMT are discussed in Subsection 4.A, and those of the vector EMT are discussed in Subsection 4.B. Ag nanoparticulate composite in an $\varepsilon_{m}=7$ dielectric is used as a model system. A discussion of the conditions under which the effective permittivity is resonant is presented in Subsections 4.A and 4.B. Effects of particle radius are also discussed in $\overline{\text { Subsec- }}$ tion $\underline{4 . B}$. Subsection $\underline{4 . \mathrm{C}}$ contains a discussion of Fano resonance that results from particle-effective-medium coupling. Conclusions are offered in Section 5 .

\section{ENSEMBLE-AVERAGED MAXWELL EQUATION}

We consider a random monodisperse, nonoverlapping spherical particulate composite in which the electrical permittivity of the matrix is assumed to be real, positive, and constant, while that of the plasmonic particles is complex and frequency-dependent. Further, as mentioned in the introduction, we assume the magnetic permeabilities of the matrix and the particle phase to be equal to that of vacuum; i.e., $\mu_{m}=$ $\mu_{p}=\mu_{0}$. This assumption is justified for dielectric matrices such as glass or water and particles of noble metals such as $\mathrm{Ag}$ or $\mathrm{Au}$. The particle diameter is assumed to be much greater than the electron mean free path in the metal. Hence, quantum confinement effects are neglected. The embedding medium is isotropic and homogenous and could either be a liquid or a solid phase. For such a system, ensemble-averaged Maxwell equations can be derived as described below.

Time-harmonic electric and magnetic fields in a source-free homogenous medium satisfy Maxwell's wave equations. For the matrix, these equations are given by

$$
\nabla \times \mathbf{E}_{m}=i \omega \mu_{m} \mathbf{H}_{m}, \quad \nabla \times \mathbf{H}_{m}=-i \omega \varepsilon_{m} \mathbf{E}_{m},
$$

where $\mathbf{E}_{m}$ and $\mathbf{H}_{m}$ are the amplitudes of the electric and magnetic fields, respectively, $\omega$ is the frequency, $\mu_{m}$ and $\varepsilon_{m}$ are the magnetic permeability and electric permittivity, respectively, and the subscript $m$ denotes the matrix medium. Similar equations apply in the particulate phase with the subscript $m$ replaced by the particle phase subscript $p$.

To obtain a macroscopic description of a random composite, we must first obtain ensemble-averaged equations. Let $g_{p}$ denote an indicator function for the particle phase whose value at a point $\mathbf{x}$ is unity if that point lies inside a particle, and zero otherwise. Note that an ensemble-average of this function is equal to the volume fraction of the particles; i.e., $\left\langle g_{p}\right\rangle(\mathbf{x})=\phi$, where the angular brackets denote an ensemble-averaged quantity. The ensemble-averaged Maxwell's equations for the random composites are obtained by multiplying the Maxwell's equations for the particle phase by its indicator function $g_{p}$ and those for the matrix phase by $1-g_{p}$, and adding the two resulting equations:

$$
\begin{gathered}
\nabla \times\langle\mathbf{E}\rangle+\left\langle\nabla g_{p} \times\left(\mathbf{E}_{m}-\mathbf{E}_{p}\right)\right\rangle=i \omega \mu_{0}\langle\mathbf{H}\rangle, \\
\nabla \times\langle\mathbf{H}\rangle+\left\langle\nabla g_{p} \times\left(\mathbf{H}_{m}-\mathbf{H}_{p}\right)\right\rangle=-i \omega\left[\varepsilon_{m}\langle\mathbf{E}\rangle+\left(\varepsilon_{p}-\varepsilon_{m}\right)\left\langle g_{p} \mathbf{E}\right\rangle\right] .
\end{gathered}
$$

Note that $\nabla g_{p}$ is zero at all points except at the matrixparticle interface where it is directed along the normal to the interface. Hence, its cross product with the difference in $\mathbf{E}$ or $\mathbf{H}$ across the interface is zero due to the fact that the tangential components of the electric and magnetic fields are continuous across the interface. Therefore, the second terms on the lefthand side of Eqs. (2a) and (2b) vanish. The term in square brackets on the right-hand side of Eq. (2b) represents the averaged electric displacement $\langle\mathbf{D}\rangle$ in the medium. We let $\langle\mathbf{D}\rangle=$ $\varepsilon_{\text {eff }}\langle\mathbf{E}\rangle$. Hence, the effective permittivity can be defined as

$$
\varepsilon_{\text {eff }}\langle\mathbf{E}\rangle=\varepsilon_{m}\langle\mathbf{E}\rangle+\left(\varepsilon_{p}-\varepsilon_{m}\right)\left\langle g_{p} \mathbf{E}\right\rangle .
$$

The wavenumber, defined as $k_{v}^{2}=\omega^{2} \mu_{v} \varepsilon_{v}, v=p, m$, also obeys Eq. (3). Hence, the effective wavenumber is given by $k_{\text {eff }}^{2}=\omega^{2} \mu_{0} \varepsilon_{\text {eff }}$. Equations (2a),$(\underline{2 b})$, and (3) can be combined using a curl operation on Eq. (2a). The resulting $\nabla \times \nabla \times\langle\mathbf{E}\rangle$ term can be shown to be equal to $-\nabla^{2}\langle\mathbf{E}\rangle$ because $\nabla$. $(\nabla\langle\mathbf{E}\rangle)=\mathbf{0}$ in the absence of free charge.

The averaged field inside the particles, given by $\left\langle g_{p} \mathbf{E}\right\rangle$, is an unknown quantity defined as

$$
\left\langle g_{p} \mathbf{E}\right\rangle(\mathbf{r}) \equiv \int_{\left|\mathbf{r}-\mathbf{r}_{1}\right| \leq a}\left\langle\mathbf{E}(\mathbf{r}) \mid \mathbf{r}_{1}\right\rangle P\left(\mathbf{r}_{1}\right) \mathrm{d} V_{\mathbf{r}_{1}} .
$$

Here, $P\left(\mathbf{r}_{1}\right)=3 \phi / 4 \pi \alpha^{3}$ is the probability of finding a particle at $\mathbf{r}_{1}, \phi$ is the particle volume fraction, and $\left\langle\mathbf{E}(\mathbf{r}) \mid \mathbf{r}_{1}\right\rangle$ is the conditionally averaged electric field. Since the governing equations are linear and the medium is overall assumed to be macroscopically isotropic, $\left\langle g_{p} \mathbf{E}\right\rangle$ can be expressed as

$$
\left\langle g_{p} \mathbf{E}\right\rangle(\mathbf{r})=\Omega\left(\phi, k_{\mathrm{eff}}\right) \phi\langle\mathbf{E}\rangle(\mathbf{r})
$$

where $\Omega$ is a constant that depends on $k_{\text {eff }}, \phi$, and the microstructure. Combination of Eqs. (3) and (5) gives $\varepsilon_{\text {eff }}$ or equivalently $k_{\text {eff }}$ as follows: 


$$
k_{\text {eff }}^{2}=k_{m}^{2}-\left(k_{p}^{2}-k_{m}^{2}\right) \Omega \phi
$$

The ensemble-averaged Maxwell equation given by

$$
\left(\nabla^{2}+k_{\text {eff }}^{2}\right)\langle\mathbf{E}\rangle=\mathbf{0}
$$

is obtained by eliminating $\mathbf{H}$ from Eqs. (2a) and (2b) followed by a substitution of $k_{\text {eff }}^{2}$ from Eq. (ㅁ). $\Omega$ can be evaluated by solving Eq. (7) followed by substitution of $\langle\mathbf{E}\rangle$ and $\left\langle g_{p} \mathbf{E}\right\rangle$ into Eqs. (5) and (6). However, because $\Omega$ is a function of $k_{\text {eff }}$, Eqs. (ㅁ) and (ㅁ) need to be solved by an iterative numerical method for the evaluation of the zeros of the following function:

$$
\Lambda\left(k_{\text {eff }}^{2}\right)=k_{\text {eff }}^{2}-k_{m}^{2}-\phi\left(k_{p}^{2}-k_{m}^{2}\right) \Omega\left(k_{\text {eff }}^{2}\right)=0 .
$$

\section{A. The Effective-Medium Model}

To determine $\Omega$, we must determine the conditionally averaged $\mathbf{E}$ with one particle fixed and then evaluate the integral in Eq. (4). We shall use an effective-medium model that has been shown to provide excellent predictions, consistent with rigorous computations that take into account multiparticle interactions of the conditionally averaged field and effective properties such as elastic moduli, attenuation and speed of acoustic waves, hydraulic permeability, effective viscosity, and particle diffusivity in suspensions [33-38]. As illustrated in Fig. 1, in this model, the conditionally averaged fields satisfy the governing equations for the suspending medium up to a distance $R$ from the center of the particle and the governing equations for the effective medium beyond that distance. $R$ is related to the static structure factor $S(\mathbf{0})$ as

$$
\frac{R}{a}=\kappa=\left(\frac{1-S(\mathbf{0})}{\phi}\right)^{\frac{1}{3}}
$$

where

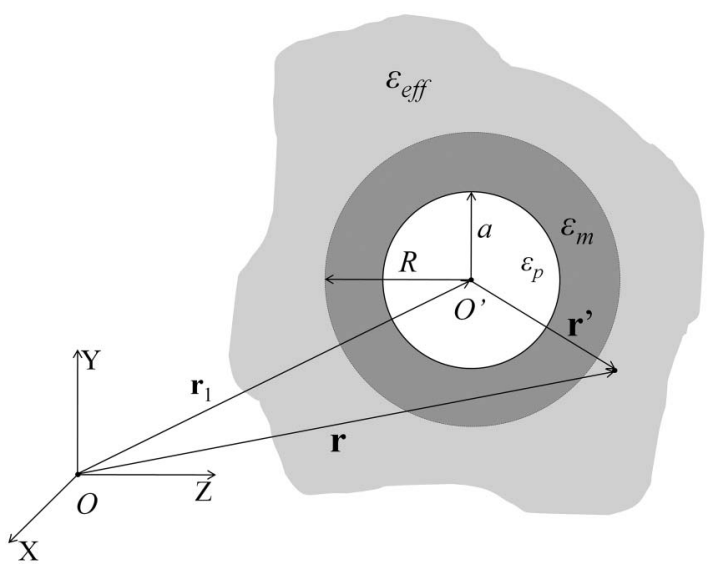

Fig. 1. A schematic of the geometry considered for the EMT. The problem of finding the conditionally averaged field in a random medium was approximated with the problem of calculating the fields in this geometry. As $r \rightarrow \infty,\langle\mathbf{E}\rangle(\mathbf{r})=\hat{\mathbf{x}} \exp \left(i k_{\mathrm{eff}} z\right)$. The unconditionally averaged wave is assumed to be $X$-polarized in the present analysis. The choice of $\mathbf{r}_{1}$ is arbitrary for a given origin $O$.

$$
S(\mathbf{0}) \equiv \int_{0}^{\infty}\left[P\left(\mathbf{r} \mid \mathbf{r}_{1}\right)-P\left(\mathbf{r}_{1}\right)\right] \mathrm{d} \mathbf{r}
$$

In Eq. $(\underline{10}), P\left(\mathbf{r} \mid \mathbf{r}_{1}\right)$ is the conditional probability density of the spheres. The quantity $S(\boldsymbol{0})$ can be interpreted as the integral of excess probability with respect to the uniform distribution. It needs to be accounted for if the random medium is to be replaced with a homogenous effective medium. In Fig. 1, $O$ denotes the origin and $O^{\prime}$ denotes the center of the particle, and the particle is located at $\mathbf{r}_{1}$. Position vectors $\mathbf{r}^{\prime}$ originate at $O^{\prime}$ such that $\mathbf{r}=\mathbf{r}_{1}+\mathbf{r}^{\prime}$.

As pointed out by Dodd et al. [35], the above choice of $R$ is necessary to ensure that the conditionally averaged field has the correct behavior at large distances from the test particle for the problem of determining the averaged diffusivity of integral membrane proteins. Subsequent studies showed that the above choice also yields excellent estimates of the effective properties even when it has no rigorous basis (see, e.g., $[\underline{33}, \underline{39}])$. Random suspensions with a hard-sphere potential have a nonzero $S(\mathbf{0})$ even in the dilute limit, which is accurately given by the Carnahan-Starling approximation [40] as

$$
S(\mathbf{0})=\frac{(1-\phi)^{4}}{1+4 \phi+4 \phi^{2}-4 \phi^{3}+\phi^{4}} .
$$

As $\phi \rightarrow 0, \kappa=2-\frac{3}{2} \phi+O\left(\phi^{2}\right)$. On the other hand, wellseparated dilute random arrays [41] have $P\left(\mathbf{r} \mid \mathbf{r}_{1}\right)=0$ for $2<r^{\prime}<\phi^{-1 / 3}, S(\mathbf{0})=0$ and hence, $\bar{\kappa}=\phi^{-1 / 3}$. We study both cases to elucidate the differences between them. Our EMT is used to evaluate $\left\langle\mathbf{E}(\mathbf{r}) \mid \mathbf{r}_{1}\right\rangle$ in Eq. (뜨).

\section{SOLUTION TECHNIQUE}

This section is devoted to a discussion on the determination of $\left\langle\mathbf{E}(\mathbf{r}) \mid \mathbf{r}_{1}\right\rangle$ for $r^{\prime}<a$, and, subsequently, the numerical calculation of $\varepsilon_{\text {eff }}$. We first show that for composites with spheres that are small compared to the wavelength of the exciting light (or equivalently, as $k a \rightarrow 0$ ), $\left\langle\mathbf{E}(\mathbf{r}) \mid \mathbf{r}_{1}\right\rangle$, and hence $\varepsilon_{\text {eff }}$ can be determined analytically by solving the electrostatic Maxwell equations (which is equivalent to setting $k a=0$ ). Further, we show that both the electrostatic approximation and Maxwell wave equations can be reduced to the Laplace equation for a scalar potential in the limit as $k a \rightarrow 0$. However, the boundary conditions for these two problems are different from each other. Since only a scalar potential is necessary to describe a static $\mathbf{E}$ field, the resulting EMT is referred to as the "scalar EMT." Thereafter, an iterative method for the numerical evaluation of $\varepsilon_{\text {eff }}$ using Maxwell wave equations for arbitrary large values of $k a$ is outlined. EMT based on Maxwell wave equations is referred to as the "vector EMT," as it involves solution of a vector Helmholtz equation. The boundary conditions for these two problems differ from each other as explained below.

\section{A. Scalar EMT}

In this section, we will show that in the limit as $k a \rightarrow 0$, Maxwell wave equations given by $\left(\nabla^{2}+k^{2}\right) \mathbf{E}=\mathbf{0}$ can be approximated by the electrostatic Maxwell equation given by $\nabla$. $\mathbf{E}_{S}=0$ for which $k a \equiv 0$, even though these equations require different boundary conditions. The electrostatic approximation is valid only for problems with a spherical symmetry 
and hence is not applicable to systems involving multiple spheres or nonspherical particles.

The static electric field $\mathbf{E}_{S}$ obeys $\nabla \cdot \mathbf{E}_{S}=0$; hence, it can be represented as the gradient of a scalar potential $\Theta$ such that $\mathbf{E}_{s}=\nabla \Theta$. Therefore, $\nabla^{2} \Theta=0$. Across an interface containing no free charge, $\Theta$ and the electric displacement $\mathbf{D}_{s} \equiv$ $\varepsilon \mathbf{E}_{s}$ are continuous. Therefore, across a spherical interface between the particle and the medium shown in Fig. 1,

$$
\Theta_{p}=\Theta_{m}
$$

and

$$
\left.\varepsilon_{p} \frac{\partial \Theta}{\partial r}\right|_{p}=\left.\varepsilon_{m} \frac{\partial \Theta}{\partial r}\right|_{m} .
$$

At an interface with no free charge and current, $\mathbf{E}$ and $\mathbf{H}$ are required to have continuous tangential components. A comparative analysis can be performed by decomposing $\mathbf{E}$ and $\mathbf{H}$ into toroidal $\Psi$ and poloidal $\Phi$ scalar potentials in the following way [33, $\underline{41-42}]$. Let

$$
\mathbf{E}=\nabla \times(\mathbf{r} \Psi)+\nabla \times \nabla \times(\mathbf{r} \Phi)
$$

and

$$
\mathbf{H}=-i \omega \varepsilon \nabla \times(\mathbf{r} \Phi)+\frac{1}{i \omega \mu_{0}} \nabla \times \nabla \times(\mathbf{r} \Psi)
$$

where $\Psi$ and $\Phi$ are solutions to the scalar Helmholtz equation. Tangential components $E_{\theta}, E_{\phi}, H_{\theta}$, and $H_{\phi}$ can be expressed as follows [는 $\underline{43}$ ]:

$$
\begin{aligned}
E_{\theta} & =\frac{1}{\sin \theta} \frac{\partial \Psi}{\partial \phi}+\frac{1}{r} \frac{\partial^{2}(r \Phi)}{\partial r \partial \theta}, \\
E_{\phi} & =\frac{\partial \Psi}{\partial \theta}+\frac{1}{r} \frac{\partial^{2}(r \Phi)}{\partial r \partial \phi}, \\
H_{\theta} & =\frac{-i \omega \varepsilon}{\sin \theta} \frac{\partial \Phi}{\partial \phi}+\frac{1}{i \omega \mu_{0} r} \frac{\partial^{2}(r \Psi)}{\partial r \partial \theta},
\end{aligned}
$$

and

$$
H_{\phi}=i \omega \varepsilon \frac{\partial \Phi}{\partial \theta}+\frac{1}{i \omega \mu_{0} r} \frac{\partial^{2}(r \Psi)}{\partial r \partial \phi}
$$

Continuity of the above tangential components of $\mathbf{E}$ and $\mathbf{H}$ at the interface necessitate that

$$
\begin{gathered}
\Psi_{p}=\Psi_{m}, \\
\left.\frac{\partial}{\partial r}(r \Psi)\right|_{p}=\left.\frac{\partial}{\partial r}(r \Psi)\right|_{m}, \\
\varepsilon_{p} \Phi_{p}=\varepsilon_{m} \Phi_{m},
\end{gathered}
$$

and

$$
\left.\frac{\partial}{\partial r}(r \Phi)\right|_{p}=\left.\frac{\partial}{\partial r}(r \Phi)\right|_{m} .
$$

As $k a \rightarrow 0, \nabla^{2} \Psi=0$ and $\nabla^{2} \Phi=0$. For a nonmagnetic system, $\Psi$ is an indeterminable constant that does not contribute to $\mathbf{E}$ as seen from Eq. (13a). Hence, although the governing equations for the electrostatic and wave problems are identical, their boundary conditions differ from each other.

As discussed in Subsection 2.A, our EMT is based on estimating the conditionally averaged fields using an effectivemedium model shown in Fig. 1. Hence, $\left\langle\Theta(\mathbf{r}) \mid \mathbf{r}_{1}\right\rangle$ and $\left\langle\Phi(\mathbf{r}) \mid \mathbf{r}_{1}\right\rangle$ are obtained by the solution of Laplace equations for $\Theta$ and $\Phi$ subject to the boundary conditions given in Eqs. (13) and (15), respectively. In order to solve the Laplace problem, the unconditionally averaged far-field given by $\langle\mathbf{E}\rangle=\hat{\mathbf{x}} \exp \left(i \mathbf{k}_{\text {eff }} \cdot \mathbf{r}\right)$ can be expressed in terms of the first Laplace harmonic in the following way. One may consider rotating the coordinate system in Fig. 1 about the $y$-axis such that $\hat{\mathbf{x}}$ is replaced by $\hat{\mathbf{y}}$ and hence $\mathbf{E}$ points in the direction of the zenith. Since $\mathbf{r}=\mathbf{r}_{1}+\mathbf{r}^{\prime}$, $\exp \left(i \mathbf{k}_{\mathrm{eff}} \cdot \mathbf{r}\right) \rightarrow \exp \left(i \mathbf{k}_{\mathrm{eff}} \cdot \mathbf{r}_{1}\right)$ as $r^{\prime} \rightarrow 0$. Hence, far-field scalar potentials $\langle\Theta\rangle$ and $\langle\Phi\rangle=\exp \left(i \mathbf{k}_{\mathrm{eff}} \cdot \mathbf{r}_{1}\right) r^{\prime} \cos \theta \cdot\left\langle\mathbf{E}(\mathbf{r}) \mid \mathbf{r}_{1}\right\rangle$ and $\left\langle\mathbf{E}_{S}(\mathbf{r}) \mid \mathbf{r}_{1}\right\rangle$ are given by $\nabla\left\langle\Theta(\mathbf{r}) \mid \mathbf{r}_{1}\right\rangle$ and $\nabla\left\langle\Phi(\mathbf{r}) \mid \mathbf{r}_{1}\right\rangle$, respectively. We find that the coefficient of the first regular harmonic is identical for $\Theta$ and $\Phi$ and consequently, $\left\langle\mathbf{E}(\mathbf{r}) \mid \mathbf{r}_{1}\right\rangle=$ $\left\langle\mathbf{E}_{S}(\mathbf{r}) \mid \mathbf{r}_{1}\right\rangle$

$$
=\exp \left(i \mathbf{k}_{\mathrm{eff}} \cdot \mathbf{r}_{1}\right) \frac{3 \varepsilon_{m}}{\varepsilon_{p}+2 \varepsilon_{m}} \hat{\mathbf{z}}, r^{\prime}<a
$$

for a sphere in an infinite matrix. A similar procedure can be employed to show that

$$
\begin{aligned}
& \left\langle\mathbf{E}_{S}(\mathbf{r}) \mid \mathbf{r}_{1}\right\rangle=\exp \left(i \mathbf{k}_{\mathrm{eff}} \cdot \mathbf{r}_{1}\right) \\
& \quad \frac{9 \varepsilon_{m} \varepsilon_{\mathrm{eff}}}{\left(\varepsilon_{p}+2 \varepsilon_{m}\right)\left(2 \varepsilon_{\mathrm{eff}}+\varepsilon_{m}\right)-2 \kappa^{-3}\left(\varepsilon_{p}+\varepsilon_{m}\right)\left(\varepsilon_{\mathrm{eff}}+\varepsilon_{m}\right)} \hat{\mathbf{z}}, r^{\prime}<a
\end{aligned}
$$

for a sphere in an effective medium shown in Fig. 1. The exponential term in Eqs. (16) and (17) is a phase factor that depends on the location of the sphere. Equations (4),$(5)$, and (6) can be used in that order to obtain an expression for $\varepsilon_{\text {eff }}$. Required algebraic manipulations are discussed in Subsection 3.B below. Exact expressions for $\varepsilon_{\text {eff }}$ are presented in Subsection 4.A.

\section{B. Vector EMT}

The determination of $\left\langle\mathbf{E}(\mathbf{r}) \mid \mathbf{r}_{1}\right\rangle$ for a finitely large value of $k a$ requires the solution of the vector Helmholtz equation for a 2-layer sphere geometry shown in Fig. 1. E inside a particle can be found by utilizing a multipole expansion. The solution given by Hightower and Richardson [43] was adapted here to obtain the following relations for $\left\langle\mathbf{E}\left(\overline{\mathbf{r})}\left|\mathbf{r}_{1}\right\rangle\right.\right.$ for $r^{\prime}<a$ :

$$
\begin{aligned}
\left\langle E_{r}(\mathbf{r}) \mid \mathbf{r}_{1}\right\rangle= & \exp \left(i \mathbf{k}_{\mathrm{eff}} \cdot \mathbf{r}_{1}\right) \frac{-i \sin \theta^{\prime} \cos \phi^{\prime}}{k_{p}^{2} r^{\prime 2}} \\
& \sum_{n=1}^{\infty} d_{n} i^{n}(2 n+1) \pi_{n}\left(\theta^{\prime}\right) \psi_{n}\left(k_{p} r^{\prime}\right),
\end{aligned}
$$




$$
\begin{aligned}
\left\langle E_{\theta}(\mathbf{r}) \mid \mathbf{r}_{1}\right\rangle= & \exp \left(i \mathbf{k}_{\mathrm{eff}} \cdot \mathbf{r}_{1}\right) \frac{\cos \phi^{\prime}}{k_{p} r^{\prime}} \\
& \times \sum_{n=1}^{\infty} i^{n} \frac{(2 n+1)}{n(n+1)}\left[c_{n} \pi_{n}\left(\theta^{\prime}\right) \psi_{n}\left(k_{p} r^{\prime}\right)\right. \\
& \left.-i d_{n} \tau_{n}\left(\theta^{\prime}\right) \psi_{n}^{\prime}\left(k_{p} r^{\prime}\right)\right]
\end{aligned}
$$

and

$$
\begin{aligned}
\left\langle E_{\phi}(\mathbf{r}) \mid \mathbf{r}_{1}\right\rangle= & \exp \left(i \mathbf{k}_{\mathrm{eff}} \cdot \mathbf{r}_{1}\right) \frac{\sin \phi^{\prime}}{k_{p} r^{\prime}} \\
& \times \sum_{n=1}^{\infty}-i^{n} \frac{(2 n+1)}{n(n+1)}\left[c_{n} \tau_{n}\left(\theta^{\prime}\right) \psi_{n}\left(k_{p} r^{\prime}\right)\right. \\
& \left.-i d_{n} \pi_{n}\left(\theta^{\prime}\right) \psi_{n}^{\prime}\left(k_{p} r^{\prime}\right)\right] .
\end{aligned}
$$

In Eqs. (18a)-(18c), primed coordinates $\left(r^{\prime}, \theta^{\prime}, \phi^{\prime}\right)$ are with respect to the origin $O^{\prime}, n$ is the order of the multipole, $\pi_{n}=P_{n}^{1}(\cos \theta) / \sin \theta$ and $\tau_{n}=d P_{n}^{1}(\cos \theta) / d \theta$ are the polar angle dependent functions related to the associated Legendre polynomials $P_{n}^{1}$ of degree $1, \psi_{n}(z) \equiv z j_{n}(z)$ are Riccati-Bessel functions associated with the spherical Bessel functions $j_{n}(z)$, and $c_{n}$ and $d_{n}$ are the corresponding Mie coefficients [21]. The expressions of $c_{n}$ and $d_{n}$ are given in Appendix $\underline{\mathrm{A}}$. In general, both $c_{n}$ and $d_{n}$ are functions of $a$ and $k_{\text {eff }}$ [21].

Bessel functions and their derivatives were calculated using established iterative techniques [44]. The volume integral of $\left\langle\mathbf{E}(\mathbf{r}) \mid \mathbf{r}_{1}\right\rangle$ over the particle volume was determined to evaluate $\left\langle g_{p} \mathbf{E}\right\rangle$ and subsequently $\Omega$ using Eqs. (4) and (5). Phase factors $\exp \left(i \mathbf{k}_{\text {eff }} \cdot \mathbf{r}_{1}\right)$ in Eqs. (18) were $\operatorname{expressed~as~} \exp \left(i \mathbf{k}_{\text {eff }} \cdot \mathbf{r}\right)$ $\exp \left(-i \mathbf{k}_{\text {eff }} \cdot \mathbf{r}^{\prime}\right)$ so that $\exp \left(i \mathbf{k}_{\text {eff }} \cdot \mathbf{r}\right)$ in the right-hand side of Eq. (5) would cancel out with the left-hand side. A two dimensional composite Simpson's rule [45] was used since the analytical evaluation of the integral in Eq. (4) over $r$ and $\theta$ was not possible due to the presence of the exponential term $\exp \left(-i \mathbf{k}_{\text {eff }} \cdot \mathbf{r}^{\prime}\right)$. Only the $x$-component of $\left\langle g_{p} \mathbf{E}\right\rangle$ was found to be nonzero, consistent with the isotropic nature of the effective medium. $k_{\text {eff }}$ and equivalently $\varepsilon_{\text {eff }}$ were calculated by finding the zeros of $\Lambda$ in Eq. (7) using Newton-Raphson iterations. Since $\Lambda$ was not necessarily analytic in the complex variable $k_{\text {eff }}$, it was treated as a function of two variables, which were the real and imaginary parts of $k_{\text {eff }}$ necessitating the use of a two dimensional Newton-Raphson method [46]. For large $\phi$, Eq. (8) permitted multiple solutions that were close to one other. Hence, the solution corresponding to the limit as $\phi \rightarrow 0$ was traced by using zero order continuation. The procedure was repeated for $\lambda$ values in the visible range (300-800 nm). Permittivity data for noble metals was obtained from [47]. All computations were performed using MATLAB.

\section{RESULTS AND DISCUSSION}

\section{A. Scalar EMT}

In the limit as $\phi \rightarrow 0, \varepsilon_{\text {eff }} \rightarrow \varepsilon_{m}$. Hence, due to the absence of particle-effective-medium coupling, $\left\langle\mathbf{E}(\mathbf{r}) \mid \mathbf{r}_{1}\right\rangle$ is given by Eq. (16). The corresponding $\varepsilon_{\text {eff }}$ is given by the following equation:

$$
\varepsilon_{\text {eff }} / \varepsilon_{m}=1+3 \beta \phi, \quad \beta=\left(\frac{\varepsilon_{p}-\varepsilon_{m}}{\varepsilon_{p}+2 \varepsilon_{m}}\right) .
$$

Here, $\beta$ is the electric polarizability per unit volume for a small sphere. As will be shown later in this section, the linear dependence shown in Eq. (19) is a general result that is independent of the microstructure since $\beta$ is a material property. In the case of a finitely large $\phi$ and arbitrary $\kappa,\left\langle\mathbf{E}(\mathbf{r}) \mid \mathbf{r}_{1}\right\rangle$ is given by Eq. (17), for which $\varepsilon_{\text {eff }}$ is found to be the following:

$$
\varepsilon_{\mathrm{eff}} / \varepsilon_{m}=1+\frac{9 \varepsilon_{\mathrm{eff}}}{\left(2 \varepsilon_{\mathrm{eff}}+\varepsilon_{m}\right)-2 \kappa^{-3} \beta\left(\varepsilon_{\mathrm{eff}}-\varepsilon_{m}\right)} \gamma, \gamma \equiv \beta \phi .
$$

For a well-separated system, $\kappa=\phi^{-1 / 3}$, which in conjunction with Eq. (20) gives the following result:

$$
\varepsilon_{\text {eff }} / \varepsilon_{m}=\frac{1+2 \gamma}{1-\gamma}=1+3 \gamma+3 \gamma^{2}+O\left(\gamma^{3}\right)
$$

Equation (21) is identical to the classical MGT, which is also a scalar EMT in which the presence of other particles is accounted through the modification of the averaged far-field $[28,48]$. The lower bound, $\kappa=1$, can be substituted in Eq. (20) to give the well-known Bruggeman mixing rule (BMR):

$$
\phi\left(\frac{\varepsilon_{p}-\varepsilon_{\text {eff }}}{\varepsilon_{p}+2 \varepsilon_{\text {eff }}}\right)+(1-\phi)\left(\frac{\varepsilon_{m}-\varepsilon_{\text {eff }}}{\varepsilon_{m}+2 \varepsilon_{\text {eff }}}\right)=0,
$$

which is based on a symmetric mixing approach for the inclusions and matrix phase. As a consequence, BMR can model percolation effects [27]. A similar concentric-shell model with a variable shell thickness was also proposed by Hashin and Shtrikman [27]. However, the dependence of the shell thickness parameter $\kappa$ on the microstructure was not demonstrated. MGT and BMR can be seen as the upper and lower Hashin-Strikman bounds of the scalar EMT. Garcia et al., among others, have derived self-consistent mixing rules for ternary plasmonic composites based on Hashin-Strikman formalism [49]. Within the framework of the EMT presented in this work, $\kappa$ is a physical parameter that can be determined from the structure factor (or equivalently the radial distribution function) of the composite. Conversely, if $\kappa$ were to be determined by fitting spectroscopic data to the EMT predictions, it can be used to glean microstructure information regarding the distribution of particles within the composite.

For a random system, $\kappa$ can be expanded about $\phi=0$ using Eqs. (9) and (11) to give $\kappa=2-\frac{4}{3} \phi+O\left(\phi^{2}\right)$. This can be substituted in Eq. (20) to obtain the following expansion for $\varepsilon_{\text {eff }}$ valid for $\gamma \ll 1$ :

$$
\varepsilon_{\mathrm{eff}} / \varepsilon_{m}=1+3 \gamma+\frac{3}{4}(\beta+4) \gamma^{2}+O\left(\gamma^{3}\right)
$$

Note that all scalar EMTs based on Eq. (20) indeed yield $\varepsilon_{\text {eff }}=\varepsilon_{p}$ for $\phi=1$.

\section{Resonance conditions}

A particle undergoes an electric resonance when $\left\langle\mathbf{E}(\mathbf{r}) \mid \mathbf{r}_{1}\right\rangle$ is singular. For a finitely large $\phi$, however, a different resonance condition that takes into account the particle-effectivemedium coupling effect will result. Hence, in this section, a discussion on the conditions under which scalar $\varepsilon_{\text {eff }}$ shows a resonance is presented. 
Resonance of a single sphere requires that

$$
|\beta| \rightarrow \infty, \quad \text { or equivalently, } \quad \varepsilon_{p}=-2 \varepsilon_{m},
$$

as can seen from Eqs. (16) and (19). Presence of an effective medium, on the other hand, leads to a condition that the denominator in Eqs. (17) and (20) vanish. Hence, one obtains a resonance condition given by

$$
2 \beta\left(\frac{\varepsilon_{\mathrm{eff}}-1}{2 \varepsilon_{\mathrm{eff}}+1}\right)=\kappa^{3} .
$$

In the specific case of a well-separated system (or MGT), substitution of $\kappa=\phi^{-1 / 3}$ in Eq. (25) gives the following resonance condition:

$$
\beta \approx \frac{1}{\phi}
$$

The above equation can also be obtained by letting the denominator in Eq. (21) be zero. Similar to the way by which Eq. (26) was obtained, a substitution of $\kappa=2-\frac{4}{3} \phi+O\left(\phi^{2}\right)$ in Eq. (25) under the limit as $\gamma \rightarrow 0$ leads to the following resonance condition for random hard-sphere composites:

$$
\beta \approx \frac{2}{\sqrt{\phi}}
$$

As seen from Eqs. (26) and (27), for dense systems, the resonance wavelength is different from that of a single particle. Hence, the scaling of $\beta$ with $\phi$ depends on the microstructure of the system through the static structure factor. This trend is consistent with the reported red-shift in plasmon resonance for ion-implanted composites [13]. We note that substitution of $\kappa=1$ in Eq. (25) in the limit as $\gamma \rightarrow 0$ gives the resonance condition in the "Bruggeman limit" as $\beta \approx 1 / 2 \sqrt{\phi}$.

Since the equations for dense systems [Eqs. (17), (20), and (24)] include particle-effective-medium coupling, they are implicit in $\varepsilon_{\text {eff }}$. Conversely, the inverse problem, i.e., when $\varepsilon_{\text {eff }}$ is given and $\varepsilon_{p}$ or $\phi$ are unknown, is explicit in all cases. Hereafter, we only consider the solution of Eq. (20) that approaches unity as $\phi \rightarrow 0$.

\section{Ag plasmonic composite}

Resonant plasmonic nanospheres made of noble metals such as $\mathrm{Ag}, \mathrm{Au}$, and $\mathrm{Cu}$ have an $\varepsilon_{p}$ that has a large negative real part and a small positive imaginary part for the visible range of the electromagnetic spectrum as shown in Fig. 2. Their $\beta$ values show a resonance as a consequence. We consider Ag in our further discussion since it has the smallest $\mathfrak{\Im}\left(\varepsilon_{p}\right)$ over a broad wavelength range and hence the most prominent $\beta$. Further, a medium with a relatively large $\varepsilon_{m}$ can shift this resonance to the red region and make it more prominent. For this work we will consider $\varepsilon_{m}=7$. Semiconductors such as $\mathrm{ZnO}, \mathrm{Si}, \mathrm{TiO}_{2}$, etc. have similar values of $\varepsilon_{m}$. Values of $\beta$ for Ag spheres in an $\varepsilon_{m}=7$ medium are shown in Fig. $\underline{3}$.

The permittivity of high conductivity metals such as Ag can be evaluated approximately by using the Drude model given by $\varepsilon_{p}=1-\omega_{p}^{2} / \omega^{2}+i \omega \omega_{c}$ [50]. Here, plasma frequency $\omega_{p} \approx$ $2.321 \times 10^{15} \mathrm{~Hz}$ and collision frequency $\omega_{c} \approx 5.513 \times 10^{12} \mathrm{~Hz}$ for $\mathrm{Ag}$ in the visible range [51]. The peak in $\mathfrak{\Im}(\beta)$ occurs at the
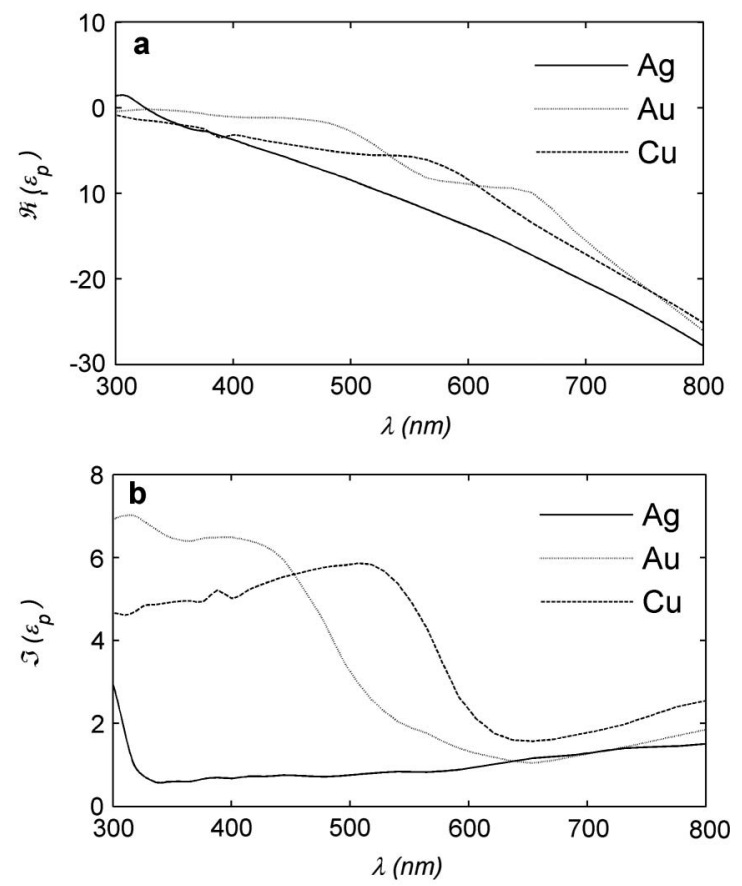

Fig. 2. Real and imaginary parts of the permittivity of high opticalconductivity metals $\mathrm{Ag}, \mathrm{Au}$, and $\mathrm{Cu}$ that are considered in this work. Permittivity data is taken from [47]. Ag has the lowest imaginary permittivity over a broad range of wavelengths.

resonance frequency given by $\omega_{R}=\frac{\omega_{p}}{\sqrt{2 \varepsilon_{m}+1}}$ or equivalently resonance wavelength $\lambda_{R}=\frac{2 \pi c \sqrt{2 \varepsilon_{m}+1}}{\omega_{p}}$, where $c$ is the speed of light in vacuum. Hence, the resonance wavelength for small spheres scales as $\sqrt{2 \varepsilon_{m}+1}$, resulting in a red-shift as $\varepsilon_{m}$ is increased. The peak value $\beta_{R}$ at resonance is given by $\beta_{R}=$ $-\left(\frac{\varepsilon_{m}-1}{2 \varepsilon_{m}+1}\right)+i \frac{\omega_{p}}{\omega_{c}} \frac{3 \varepsilon_{m}}{\left(2 \varepsilon_{m}+1\right)^{3 / 2}}$ for a Drude metal.

The real and imaginary parts of $\beta$ represent the reactive and dissipative components, respectively. At resonance $(\lambda \approx 600 \mathrm{~nm}), \mathfrak{J}(\beta)$ is a large positive number and $\mathfrak{R}(\beta)=0$, as shown in Fig. 3. As $|\gamma| \rightarrow 0$, the dissipative term for all scalar EMTs is given by $\mathfrak{J}\left(\varepsilon_{\text {eff }} / \varepsilon_{m}\right) \approx 3 \phi \mathfrak{\Im}(\beta)$. The quadratic term for random systems is $-\frac{3}{4}[\Im(\beta)]^{3} \phi^{2}$. As a result, $\mathfrak{\Im}\left(\varepsilon_{\text {eff }}\right)$ is highly nonlinear in $\phi$. For $\beta$ shown in Fig. $3, \mathfrak{J}\left(\varepsilon_{\text {eff }} / \varepsilon_{m}\right) \approx 69 \phi-$ $912525 \phi^{2}$ at resonance. In contrast, it is identically zero for

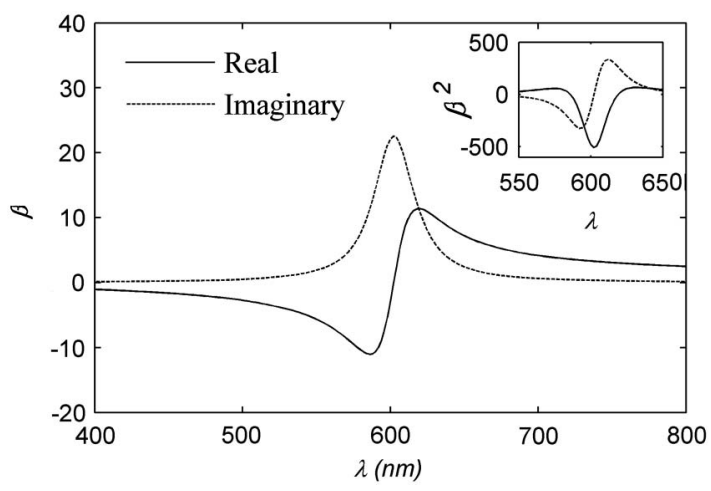

Fig. 3. $\beta$ for Ag spheres in a $\varepsilon_{m}=7$ medium. Resonance occurs for $\lambda \approx 600 \mathrm{~nm}$ and $\beta \approx 23 i . \mathfrak{J}(\beta)$ is a small number away from resonance, and $\mathfrak{R}(\beta)$ changes sign from negative to positive upon moving from blue to red regions about resonance. 


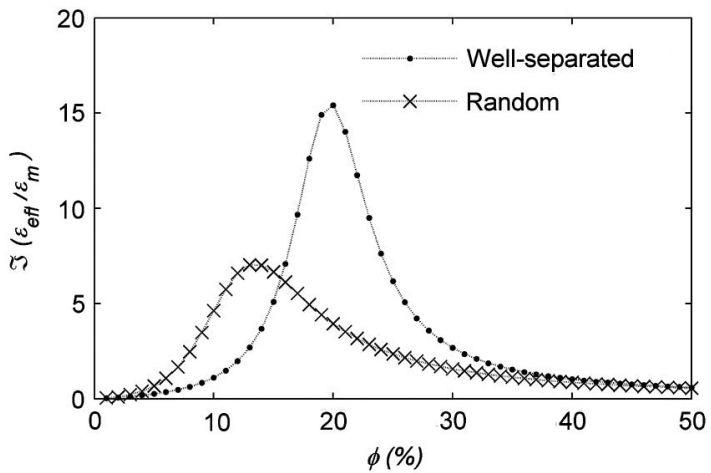

Fig. 4. $\mathfrak{J}\left(\varepsilon_{\text {eff }}\right)$ predicted by the scalar EMT for random and wellseparated microstructures. Here, $\lambda \approx 680 \mathrm{~nm}$ and $\beta=4.973+0.973 i$. $\beta$ has a resonance peak at $\lambda \approx 600 \mathrm{~nm}$, as shown in Fig. 3 .

a well-separated composite. Hence, quadratic coupling does not lead to any loss in a well-separated composite. $\mathfrak{R}\left(\varepsilon_{\mathrm{eff}} / \varepsilon_{m}\right)$ is identically one under resonance for a random system. Hence, the reaction originates only from the medium. For a well-separated system, quadratic and even powered coupling contributes to the reaction such that $\mathfrak{R}\left(\varepsilon_{\text {eff }} / \varepsilon_{m}\right) \approx$ $1-3[\Im(\beta) \phi]^{2}$.

At off-resonance, $\mathfrak{R}(\beta)$ is greater than zero for $\lambda>\lambda_{R}$ and less than zero for $\lambda<\lambda_{R}$. $\Im(\beta)$ is smaller in comparison for the most part. For instance, at $\lambda \approx 680 \mathrm{~nm}, \beta=4.973+0.973 i$, and at $\lambda \approx 520 \mathrm{~nm}, \beta=-3.546+0.816 i$. The red regions exhibit a "concentration resonance," which occurs when the resonance condition given in Eq. (24) is satisfied. In the case of a wellseparated system, the condition is $\beta \phi=1$, and for a random system, it is $\beta^{2} \phi \approx 4$, as given in Eqs. (25) and (26). Figure $\underline{4}$ shows $\mathfrak{\Im}\left(\varepsilon_{\text {eff }} / \varepsilon_{m}\right)$ as a function of $\phi$ for $\lambda \approx 680 \mathrm{~nm}$. A peak appears at $\phi \approx 13 \%$ for a random system and at $\phi \approx 20 \%$ for a well-separated system. The peak for a random system is less prominent compared to that for a well-separated system. The resonance concentration shifts to higher values of $\phi$ as $\varepsilon_{m}$ is decreased.

Figure 5 shows the effect of $\phi$ on $\varepsilon_{\text {eff }}$. It is evident that a well-separated system couples more intensely with the incident field as compared to a random one. Peaks corresponding to random systems occur for $\lambda$ values that are larger than those for well-separated systems due to the difference in the scaling of $\beta$ with respect to $\phi$ under resonance, as shown in Eqs. (26) and (27). The quadratic coefficient for a random system is $\frac{3}{4} \beta^{3}+3 \beta^{2}$ [Eq. (22)] in comparison to $3 \beta^{2}$ [Eq. (21)] for a well-separated system. The coefficients of the cubic and higher order terms in $\phi$ also depend on higher powers of $\beta$ for a random system. Hence, the resonance condition for the coefficient of the quadratic term in the power series expansion of $\varepsilon_{\text {eff }}(\phi)$ will depend on that of the linear term, while the resonance condition for cubic coefficient will depend on those of the quadratic and linear terms, etc. As a consequence, the peak in $\mathfrak{\Im}\left(\varepsilon_{\text {eff }}\right)$ for random systems is not symmetric about its maximum and is broader in comparison to that for a wellseparated system or a single sphere.

\section{B. Vector EMT}

This subsection describes the effect of $k a$ on numerically calculated values of $\varepsilon_{\text {eff }}$. An analysis of vector EMT in the limit as $\phi \rightarrow 0$ and $k a \rightarrow 0$ is first presented. Resonance analysis in the
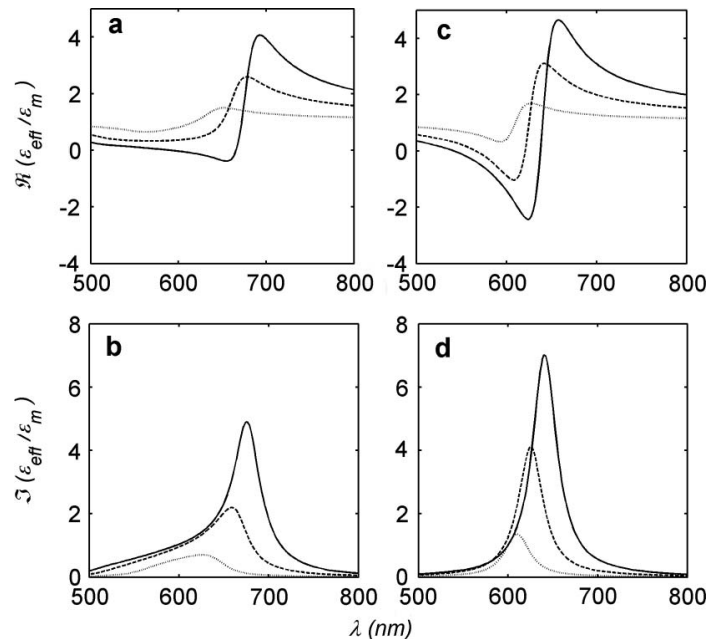

Fig. 5. $\varepsilon_{\text {eff }}$ for a composite with Ag NPs in an $\varepsilon_{m}=7$ medium calculated with the scalar EMT. Random (a) and (b) and well-separated random composites (c) and (d) for $\phi=2 \%$ (solid), 6\% (dashed), and $10 \%$ (dotted) were considered. The resonance peak is more red-shifted and broad for a random system. A well-separated system shows a more intense resonance with a symmetric peak in comparison. Stronger coupling in a random system leads to a tail in the blue region.

limit as $\phi \rightarrow 0$, followed by a discussion on the numerical results for finitely large $\phi$ and $k a$ will also be presented.

In the vector EMT, $\left\langle\mathbf{E}(\mathbf{r}) \mid \mathbf{r}_{1}\right\rangle$ is not a constant vector but depends on the toroidal (corresponding to coefficients $c_{n}$ ) and poloidal (corresponding to coefficients $d_{n}$ ) multipoles shown in Eqs. (18). Only poloidal modes are capable of generating $\left\langle E_{r} \mid \mathbf{r}_{1}\right\rangle$, which only toroidal modes generate $\left\langle H_{r} \mid \mathbf{r}_{1}\right\rangle$. Hence, we will refer to the poloidal modes as the electric modes and the toroidal modes as magnetic modes following the standard convention [42].

For subsequent analysis, the following expansion is used to express the dependence of $\varepsilon_{\text {eff }}$ on $\phi$ :

$$
\varepsilon_{\mathrm{eff}} / \varepsilon_{\mathrm{eff}}=1+A \phi+B \phi^{2}+O\left(\phi^{3}\right)
$$

As in Subsection 3.A, only the linear and quadratic coefficients are of interest here. Coefficients $A$ and $B$ in Eq. (28) depend on $k a, \varepsilon_{p}$, and $\varepsilon_{m}$. Riccati-Bessel functions in Eqs. (18) can be expanded into a Taylor series as $\psi_{n}(z)=C_{n} z^{n+1}+O\left(z^{n+3}\right)$, where $C_{n}=\frac{\sqrt{\pi}}{4 \Gamma(n+3 / 2)}$, where $\Gamma$ denotes the gamma function. Hence, as $z \rightarrow 0$ or equivalently $k_{p} a \rightarrow 0$, the terms corresponding the $c_{1}$ mode in Eqs. (18) are $O(z)$, while those corresponding to the $d_{1}$ mode are $O(1)$. As a result, only the $d_{1}$ term contributes significantly as $z \rightarrow 0$. In the limit as $\phi \rightarrow 0$, only the linear coefficient $A$ in Eq. (28) is relevant irrespective of the microstructure. $\left\langle\mathbf{E}(\mathbf{r}) \mid \mathbf{r}_{1}\right\rangle$ from Eqs. (18) can be used together with Eqs. (4) and (5) to obtain the parameter $\Omega$. It can be shown that $\Omega=d_{1}$ and as $k_{p} a \rightarrow 0$, where $d_{1}$ is given by $d_{1}=\frac{3}{\left(\varepsilon_{p}+2\right)}\left[1+i \frac{2}{3} \beta\left(k^{*} a\right)^{3}\right]+O\left[\left(k^{*} a\right)^{6}\right]$. Subsequently, $A$ can obtained by using Eq. (ㅁ) as

$$
A=3 \beta+i 2 \beta^{2}\left(k^{*} a\right)^{3}+O\left[\left(k^{*} a\right)^{6}\right],
$$

where $k^{*}=k_{p} / n_{\text {eff }}$. Note that as $\phi \rightarrow 0, n_{\text {eff }} \rightarrow n_{m}$; hence, $k^{*}$ can be modified appropriately in the dilute limit. Mallet $e t a l$. [29] have recently rederived MGT for finitely large particles 
that can exhibit scattering using rigorous Foldy-Lax multiple scattering equations (see Eq. (22) in [29]). The linear term is identical to the one obtained here in Eq. (29). The cubic dependence on the particle radius implies that the size effect becomes significant only when $\left|\beta^{2}\left(k^{*} a\right)^{3}\right|$ is $O(1)$. Since $\beta^{2}$ is a large negative number at resonance as shown in the Fig. 3 inset, size effects become significant even for relatively small radii for resonant systems. For example, $\left|\beta^{2}\right|=500$ at resonance for an Ag sphere shown in Fig. 3. Hence, $\left|\beta^{2}\left(k^{*} a\right)^{3}\right|=$ $1 / 2$ for $\left|k^{*} a\right| \approx 0.063$. Here, since $k^{*}=0.0005+0.0147 i \mathrm{~nm}^{-1}$, a relatively small radius of $a \approx 34 \mathrm{~nm}$ can significantly affect $\varepsilon_{\text {eff }}$ even in the dilute limit.

\section{Resonance conditions}

Resonance occurs when $\left\langle\mathbf{E}(\mathbf{r}) \mid \mathbf{r}_{1}\right\rangle$ given by Eqs. (18) is singular. In turn, this requires that the coefficients $c_{n}$ and $d_{n}$ are singular. Magnetic resonances represented by singular $c_{n} s$ do not occur in plasmonic systems since $\mu_{p}=\mu_{m}=\mu_{0}$. Vector EMT exhibits only electric multipole resonances that correspond to $d_{n}$ that are given in Appendix A. The conditions are complicated for Helmholtz multipoles, as they involve the Riccati-Bessel functions. However, the underlying physical aspects can be appreciated by utilizing the simpler Laplace multipoles [52]. The $n$th Laplace multipole has a size independent polarizability $\beta_{n}$ defined as

$$
\beta_{n}=\frac{\varepsilon_{p}-\varepsilon_{m}}{\varepsilon_{p}+\frac{n+1}{n} \varepsilon_{m}}
$$

A dipole resonance requires that $\beta_{1} \rightarrow \infty$ or equivalently $\varepsilon_{p}=-2 \varepsilon_{m}$; quadrupole resonance occurs when $\beta_{2} \rightarrow \infty$ or $\varepsilon_{p}=-\frac{3}{2} \varepsilon_{m}$ and so on [53]. Hence, higher order multipoles become resonant at smaller negative values of $\varepsilon_{p}$, or equivalently for smaller values of $\lambda$, as inferred from the $\varepsilon_{p}-\lambda$ curve shown in Fig. 2. Mie coefficients $A_{n}, a_{n}$, and $d_{n}$ are polynomials of $k^{*} a$ and $\beta_{n}$ in the limit as $k^{*} a \rightarrow 0$ such that higher order $\beta_{n}$ becomes significant only for an $O(1) k^{*} a[21,53]$. For an arbitrary $k^{*} a$, Helmholtz multipole electric polarizabilities $d_{n}$ depend on $k^{*} a$ [53] such that their resonance peak red-shifts with $k^{*} a$. Hence, both $A$ and $B$ red-shift as $k^{*} a$ increases.

The above mentioned physical trends in the resonance conditions are also seen in the numerical results obtained for the vector EMT. Figure 6 shows $A$ as a function of $\lambda$ for diameters $d_{p}=10,30,50$, and $100 \mathrm{~nm}$. Here, $k^{*} a \approx \frac{0.45 d_{p} \sqrt{\varepsilon_{p}}}{\lambda}$, where $d_{p}$ and $\lambda$ are in nanometers. $A$ was calculated by fitting the data for $\varepsilon_{\text {eff }}$ obtained for $\phi=0.01 \%$ to Eq. (르) as

$$
A=\frac{1}{\phi}\left(\frac{\varepsilon_{\text {eff }}}{\varepsilon_{m}}-1\right) \text {. }
$$

For a small value of $d_{p}$ such as $10 \mathrm{~nm}$, only the dipole mode is significant. Hence, $A=3 \beta$. Relatively larger particles, e.g., $d_{p}=50 \mathrm{~nm}$, show a significant quadrupole resonance. The dotted curves in Fig. 6 show a quadrupole peak at $\lambda \approx 580 \mathrm{~nm}$. A further larger $d_{p}=100 \mathrm{~nm}$ results in an octupole peak as depicted in the insets of Fig. 6 . Each peak red-shifts for larger $d_{p}$. For example, the quadrupole peak for $d_{p}=100 \mathrm{~nm}$ occurs at $\lambda \approx 650 \mathrm{~nm}$. E becomes highly localized at the particle surface for relatively large $k^{*} a$ values. Consequently, the magnitude of $A$ and $\varepsilon_{\text {eff }}$ is diminished for relatively large $d_{p}$ values as shown in Fig. $\underline{6}$. In the large size limit $\left(k^{*} a \rightarrow \infty\right), A=0$.
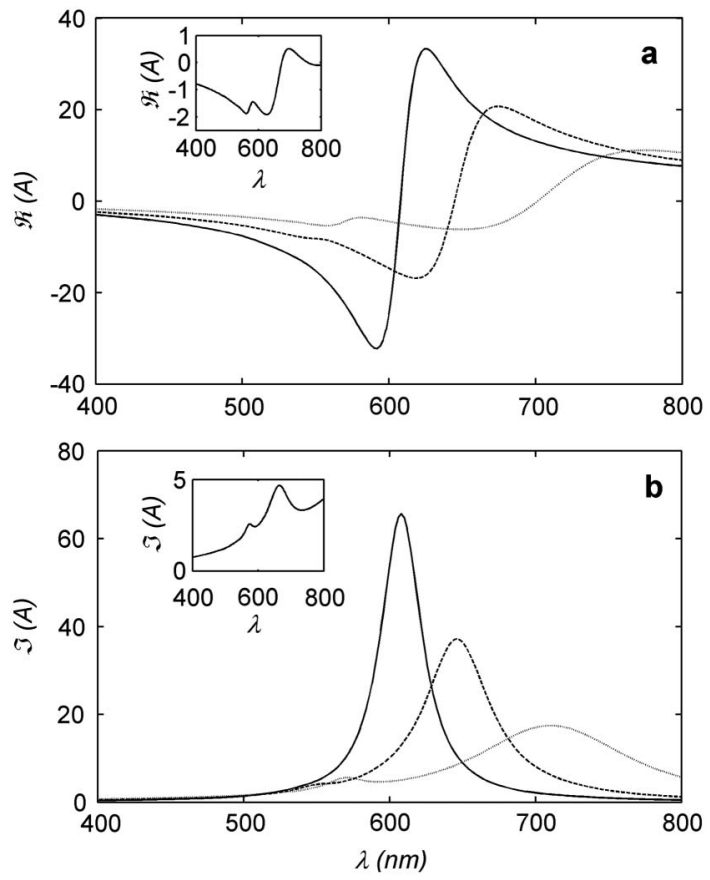

Fig. 6. Linear coefficient $A$ for composites in an $\varepsilon_{m}=7$ matrix with Ag NPs with diameters $d_{p}=10$ (solid), 30 (dashed), 50 (dotted), and $100 \mathrm{~nm}$ (inset). Here, $k^{*} a \approx \frac{0.45 d_{p} \sqrt{\varepsilon_{p}}}{\lambda}<1$ only for the blue curve. Quadrupolar and octupolar resonance peaks are present for large particles, as seen in the curves in the insets. Dipole resonance is most prominent and red-shifts as $d_{p}$ is increased. The linear coefficient becomes less significant for large particles as they screen most of the $\mathbf{E}$ field from their interior.

Further, an inspection of Eqs. (3) and (18) shows that $\varepsilon_{\text {eff }}=$ $\varepsilon_{m}$ for arbitrarily large $\phi$, in the limit as $k^{*} a \rightarrow \infty$. This is consistent with the ray optics scenario.

By neglecting cubic and higher order terms in Eq. (28), the coefficient $B$ was obtained using the following expression with $\phi=1 \%$ :

$$
B=\frac{1}{\phi^{2}}\left(\frac{\varepsilon_{\text {eff }}}{\varepsilon_{m}}-1-A \phi\right) .
$$

Figure 7 shows the values of $B$ calculated for random $[\kappa$ given by Eqs. $(9)$ and (11)] and well-separated $\left(\kappa=\phi^{-1 / 3}\right)$ random systems. $B$ represents the strength of interparticle coupling. Hence, random systems have a larger $B$. The $n$th multipole decays as $r^{-(n+2)}$ in general. Hence, dipoles can couple most strongly due to a $r^{-3}$ dependence, while higher order multipoles such as quadrupoles and octupoles couple weakly. The dotted $\left(d_{p}=30 \mathrm{~nm}\right)$ and dashed $\left(d_{p}=50 \mathrm{~nm}\right)$ curves and the solid curve in the inset $\left(d_{p}=100 \mathrm{~nm}\right)$ in Fig. 7 have a lower magnitude in comparison to the solid curve as a result. In Fig. 7(b), the solid curve for $d_{p}=10 \mathrm{~nm}$ shows a prominent radiant peak for $\lambda \approx 610 \mathrm{~nm}$ with $\Im(B) \approx-3000$. For larger particles such as the ones with $d_{p}=30 \mathrm{~nm}$ and $d_{p}=50 \mathrm{~nm}$, peak values of $\mathfrak{I}(B)$ are greatly diminished due to reduced coupling. Hence, the dashed curves in Fig. 7(b), have a peak at $\sim-900$ and the dotted curve at $\sim-100$. The corresponding $A$ values do not vary proportionately, as can be seen from Fig. 6. Hence, linear approximation is appropriate only for large $d_{p}$ values. This is not surprising since for a given $\phi$, increasing $d_{p}$ results in reducing particle number density, and 

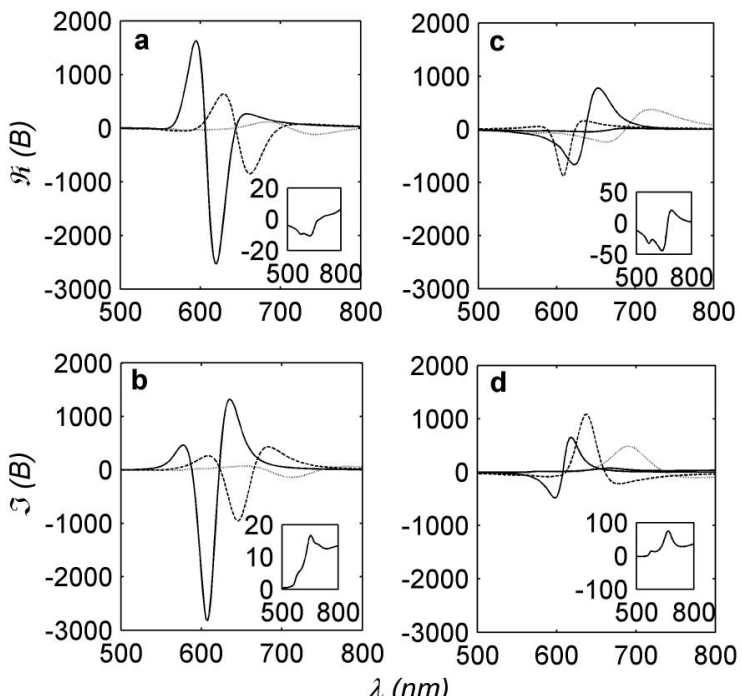

Fig. 7. Quadratic coefficient $B$ calculated for random (a) and (b) and well-separated random (c) and (d) composites in an $\varepsilon_{m}=7$ matrix containing Ag NPs with diameters $d_{p}=10$ (solid), 30 (dashed), 50 (dotted), and $100 \mathrm{~nm}$ (inset). Here, $k^{*} a \approx \frac{0.45 d_{p} \sqrt{\varepsilon_{p}}}{\lambda}<1$ is less than 1 only for the solid curve that is given by $\frac{3}{4}(\beta+4) \beta^{2}$ for (a) and (b); and $3 \beta^{2}$ for (c) and (d). Weak coupling in well-separated random systems leads to a smaller $B$ in comparison to random systems.

consequently interparticle coupling. The linear approximation is also applicable to well-separated systems since they have small values of $B$ in comparison to a random system. A maximum in $\mathfrak{J}(B)$ also occurs upon increasing $d_{p}$ while keeping $\phi$ and $\lambda$ constant, leading to a size resonance. Thus, $\varepsilon_{\text {eff }}$ exhibits resonances as a function of all system parameters.

\section{Fano Resonance}

High conductivity metals are typically described by adding to the Drude model a number of Lorentz oscillators that capture effects of lattice polarizability and interband and intraband electron transitions [54]. The Lorentz model is based on a damped harmonic oscillator with finite mass. The Drude model, however, does not include the harmonic force and hence is able to model free electrons well. The Lorentz model predicts a symmetric profile for the intensity versus frequency curve for systems with a small damping. Interestingly, $\beta$ that is based on a Drude model for $\varepsilon_{p}$ has a lineshape of a Lorentz oscillator. Hence, a single plasmonic particle is a Lorentz oscillator. In the limit as $\phi \rightarrow 0$, the susceptibility of the effective medium is given by $\chi_{\text {eff }}=\varepsilon_{\text {eff }} / \varepsilon_{m}-1=3 \beta \phi+O\left(\phi^{2}\right)$. Hence, the effective medium is also a Lorentz oscillator in the limit as $\phi \rightarrow 0$. However, the coupling between the particle and the effective medium becomes stronger; i.e., for large $\phi$ or small $\kappa, \varepsilon_{\text {eff }}$ can be expected to possess the characteristics of a coupled oscillator system that deviates from the Lorentzian symmetric lineshape. It is well-known that an unusual lineshape that is asymmetric about the extremum, known as Fano resonance, is observed in resonant coupled oscillator systems such as plasmonic nanostructures [55-58].

Within the framework of the EMT presented here, for a given $\phi, \kappa$ represents the extent of coupling between the particle and the effective medium. Consequently, $\varepsilon_{\text {eff }}-\lambda$ curves show unusual resonance shapes for relatively small values of $\kappa$. Figure 8 shows a plot of $\varepsilon_{\text {eff }}$ for Ag NP composite in an
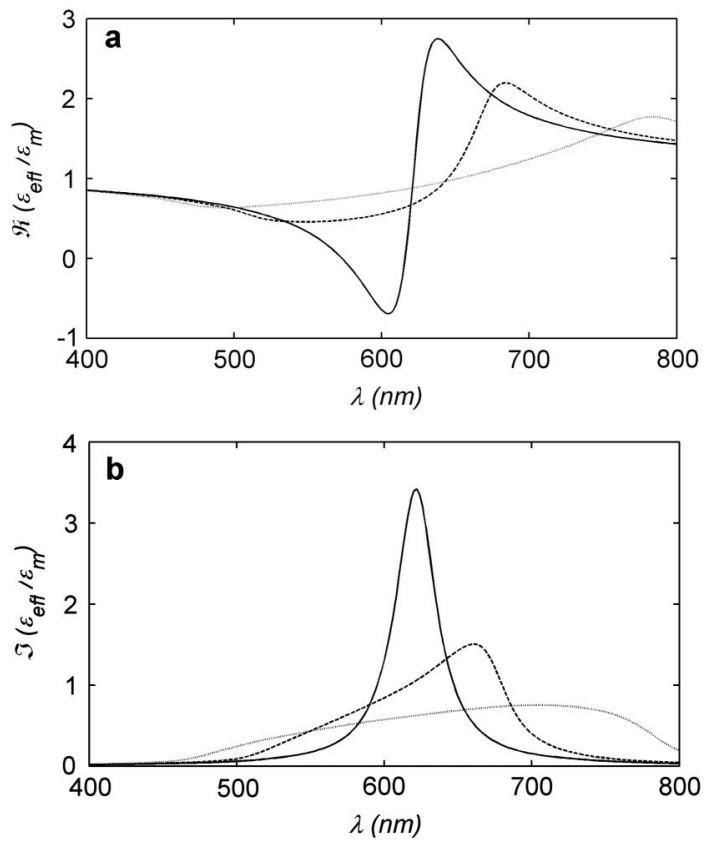

Fig. 8. Effect of $\kappa$ on $\varepsilon_{\text {eff }}$ for $\phi=5 \%$. Microstructures with $\kappa=$ $\phi^{-1 / 3} \approx 2.71$ (solid curve), $\kappa \approx 1.75$ (dashed curve), and $\kappa \approx 1.25$ (dotted curve). Small values of $\kappa$ lead to a stronger coupling that distorts the Lorentzian shape of $\varepsilon_{\text {eff }}$ even for relatively small values of $\phi$ such as $5 \%$. Calculations were performed with the scalar EMT.

$\varepsilon_{m}=7$ matrix for $\phi=5 \%$. The scalar EMT was used in the calculations. Hence, the results are representative of those for small particles. For random systems, $\kappa$ has two bounds: an upper bound given by $\kappa=\phi^{-1 / 3}$ for a system with wellseparated particles and a lower one given by $\kappa=1$, representing a locally dense composite, which we refer to as the Bruggeman limit. Close to the upper bound, the particleeffective-medium coupling is relatively weak, resulting in a symmetric Lorentzian $\varepsilon_{\text {eff }}$ even for relatively large $\phi$. This can be seen in the shape of the solid curve in Fig. $\underline{8}$. The dashed curve in Fig. 9 is the locus for the upper bound in the $\kappa-\phi$ space. Black circles in Fig. $\underline{9}$ denote the locations at which the shape of $\varepsilon_{\text {eff }}-\lambda$ curve is Lorentzian. As $\kappa$ is

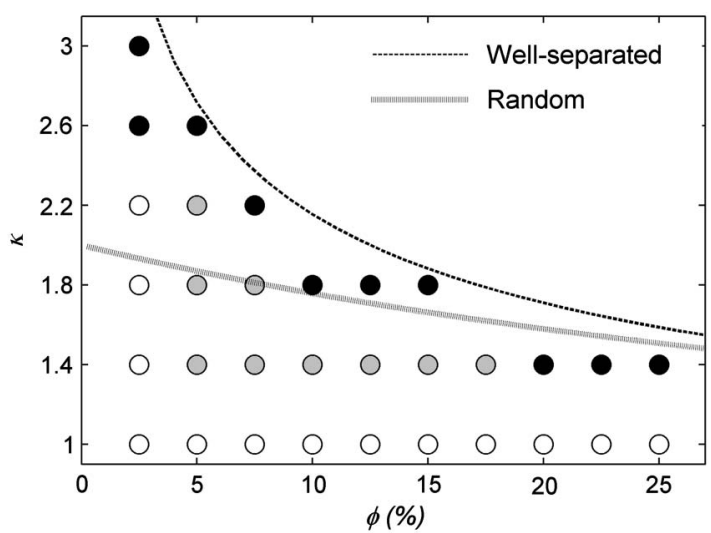

Fig. 9. Characterization of the dielectric response of a random plasmonic composite in the $\kappa-\phi$ space. The dashed line represents the upper bound for a random composite with well-separated particles, and the gray dotted line represents a random hard-sphere composite. Locations of Lorentzian and Fano responses are shown in black and gray circles, respectively. Unfilled circles denote locations in which broad lineshapes are observed. 
reduced, the shapes of the curves become distorted due to increased coupling. The resonance location shifts to larger values of $\lambda$, and the resonant peak becomes broader. $\Im\left(\varepsilon_{\text {eff }} / \varepsilon_{m}\right)$ has an asymmetric shape for intermediate values of $\kappa$, as shown in dashed curves in Fig. 8 . The $\kappa-\phi$ space locations for which an asymmetric response is predicted are shown in Fig. 9 as gray circles. For $\kappa$ values that are close to unity, $\Im\left(\varepsilon_{\text {eff }} / \bar{\varepsilon}_{m}\right)$ curve becomes very broad, as can be seen from the dotted lines in Fig. 8. Unfilled circles in Fig. 9 represent this type of response. The trends in Figs. $\underline{8}$ and $\underline{9}$ are also present in composites with a finitely large $k a$.

Fano resonance can also be understood as the interference between the absorbing and radiating modes in plasmonic structures [55,56]. Our analysis in the limit as $\phi \rightarrow 0$ suggests that the interference manifests through the Taylor coefficients of $\varepsilon_{\text {eff }}$. A negative extreme in the imaginary quadratic coefficient $\mathfrak{J}(B)$ [Figs. $\underline{7(\mathrm{~b})}$ and $\underline{7(\mathrm{~d})}$ ], for example, represents a coupled "radiating mode" that is present in conjunction with the "absorbing mode" of a single particle given by a positive $\mathfrak{\Im}(A)$ [Fig. 6b].

\section{CONCLUSIONS}

Plasmonic NPs undergo an electric resonance when their electric dipole polarizability $\beta$ is a large positive imaginary number. As a result, the particles absorb intensely. For a relatively large volume fraction $\phi$ and/or when particle size is comparable to that of the exciting wavelength, interparticle coupling becomes important. Consequently, the effective permittivity $\varepsilon_{\text {eff }}$ can no longer be treated as the sum of the polarizabilities of the individual particles and, hence, it does not vary linearly with $\phi$. Hence, quadratic and higher order effects in $\phi$ on the polarizability have to be determined to obtain accurate predictions of $\varepsilon_{\text {eff }}$.

In this work, we have developed an EMT to account for such nonlinear effects on the effective permittivity of dense random dispersions of equi-sized spheres of high opticalconductivity metals such as $\mathrm{Ag}, \mathrm{Au}$, and $\mathrm{Cu}$. The EMT is based on the idea that the region surrounding a given particle in a composite can be modeled as an effective continuum that begins after a distance $R=k a$ from the center of the particle $(\kappa>1)$. Within this framework, $\kappa$ is interpreted as a microstructure parameter that correlates with the static structure factor of the composite. For a homogenous random composite, $\kappa$ is bounded such that $1<\kappa<\phi^{-1 / 3}$. The upper bound corresponds to a well-separated random system that can be modeled as a Maxwell-Garnett composite. The lower bound corresponds to Bruggeman's mixing rule which, as in the case of MGT, is based on the electrostatic approximation [27]. In general, a random hard-sphere microstructure would have a $\kappa$ value that lies in between these two bounds, which, in principle, can be determined from the knowledge of the radial distribution function. Hence $\kappa$ is a physical rather than an adjustable fitting parameter. Conversely, if $\kappa$ were to be determined by fitting spectroscopic data to the EMT predictions, it can be used to better understand the internal microstructure of the composite.

The scalar (electrostatic) approximation is valid only for particles much smaller than the exciting wavelength. The EMT presented here takes into account both the microstructure and finite size effects in a self-consistent fashion. Specifically, two scenarios were examined, one in which $k a \rightarrow 0$ in which the conditionally averaged electric field can be obtained by the solution of the Laplace equation for the electrostatic potential (scalar EMT) and a more general case for finitely large spheres for which a solution of vector Helmholtz equation for $\mathbf{E}$ is required (vector EMT).

Resonance conditions for individual particles were found to depend on $\kappa$ and $\phi$. In the limit as $k a \rightarrow 0$, the scaling of the particle polarizability at resonance with $\phi$ depends on the microstructure. A well-separated random composite has an $\varepsilon_{\text {eff }}$ resonance when $\beta \approx 1 / \phi$. In contrast, for a random hard-sphere composite, the resonance condition is given by $\beta \approx 2 / \sqrt{\phi}$. Hence, for a given $\phi$, the resonance peak is more red-shifted for random systems. For finite sized spheres, the vector EMT problem was solved numerically to obtain the a quadratic approximation for $\varepsilon_{\text {eff }}$ as a function of $\phi$. As the particle size is increased, $\varepsilon_{\text {eff }}$ versus the $\lambda$ curve exhibits multiple peaks corresponding to quadrupolar, octupolar, and higher order resonances in addition to the dipolar resonance. Size effect on $\varepsilon_{\text {eff }}$ becomes significant when $\left|\beta^{2}\left(k^{*} a\right)^{3}\right|$ is $O(1)$. Hence, for composites consisting of high conductivity metals such as $\mathrm{Ag}$ in a medium with large refractive index in the visible range such as $\mathrm{TiO}_{2}, \mathrm{ZnO}$, and $\mathrm{Si}$, size effects could manifest even for particle diameters of a few tens of nanometers.

Particle-effective-medium coupling gives rise to a nonLorentzian resonance behavior in $\varepsilon_{\text {eff }}$. In order to characterize the resonant optical response in the $\kappa-\phi$ space, a "phase diagram" was constructed. Three regions were identified based on the lineshape of $\mathfrak{I}\left(\varepsilon_{\text {eff }}\right)$ : (i) Lorentzian (symmetric peak), (ii) Fano (distinctly asymmetric peak), and (iii) Bruggeman (broad). For an Ag NPs in a high refractive index medium, the Fano resonance region is enveloped by the Lorentzian (large $\kappa$ or large $\phi$ ) and Bruggeman (small $\kappa$ or small $\phi$ ) regions. Overall, the predictions of the EMT are in qualitative agreement with experimental trends observed for plasmonic composites [13]. This work motivates experimental investigations to quantify the effect of volume fraction on the optical response of plasmonic nanocomposites with well-characterized microstructures. As discussed in Appendix B, the EMT presented here can be extended to describe linear optical response of polydisperse and multiple species systems by adapting the methodology described by Koo and Sangani [39]. Garcia et al.'s approach for the description of nonlinear optical properties of multiple species of nanocomposites can also be incorporated to further extend the EMT [59].

\section{APPENDIX A: $c_{n}$ AND $d_{n}$}

Mie coefficients, $c_{n}$ and $d_{n}$, for $\mathbf{E}$ inside a particle in a layered sphere geometry shown in Fig. $\underline{1}$ are discussed in Section $\underline{3}$. Hightower and Richardson showed that they can be calculated in the following fashion [43]:

$c_{n}=\left[\frac{k_{p} \psi_{n}\left(k_{m} a\right)-B_{n} k_{p} \chi_{n}\left(k_{m} a\right)}{k_{m} \psi_{n}\left(k_{p} a\right)}\right]\left[\frac{k_{m} \psi_{n}\left(k_{\mathrm{eff}} R\right)-b_{n} k_{m} \zeta_{n}\left(k_{\mathrm{eff}} R\right)}{\psi_{n}\left(k_{m} R\right)-B_{n} \chi_{n}\left(k_{m} R\right)}\right]$

and

$$
d_{n}=\left[\frac{\psi_{n}\left(k_{m} a\right)-A_{n} \chi_{n}\left(k_{m} a\right)}{\psi_{n}\left(k_{p} a\right)}\right]\left[\frac{\psi_{n}\left(k_{\mathrm{eff}} R\right)-a_{n} \zeta_{n}\left(k_{\mathrm{eff}} R\right)}{\psi_{n}\left(k_{m} R\right)-A_{n} \chi_{n}\left(k_{m} R\right)}\right] .
$$

Here, $k_{\nu}, \nu=p, m$, eff denotes the wavenumber in the particle, medium, and effective medium, respectively. Particle radius is 
$a$, and $R$ is the shell radius as shown in Fig. 1. Riccati-Bessel functions $\psi_{n}, \chi_{n}$, and $\zeta_{n}$ are defined as

$$
\begin{aligned}
& \psi_{n}(z) \equiv z j_{n}(z), \\
& \chi_{n}(z) \equiv-z y_{n}(z),
\end{aligned}
$$

and

$$
\zeta_{n}(z) \equiv z h_{n}^{(1)}(z)
$$

Here, $j_{n}, y_{n}$, and $h_{n}^{(1)}$, respectively, are the regular, singular, and outward propagating spherical Bessel functions. Note that spherical Hankel functions of the first kind are defined as $h_{n}^{(1)}=j_{n}+i y_{n}$ [45]. The coefficients $A_{n}$ and $B_{n}$ have the following form:

$$
A_{n}=\frac{k_{m} \psi_{n}\left(k_{m} a\right) \psi_{n}^{\prime}\left(k_{p} a\right)-\psi_{n}^{\prime}\left(k_{m} a\right) \psi_{n}\left(k_{p} a\right)}{k_{m} \chi_{n}\left(k_{m} a\right) \psi_{n}^{\prime}\left(k_{p} a\right)-\chi_{n}^{\prime}\left(k_{m} a\right) \psi_{n}\left(k_{p} a\right)}
$$

and

$$
B_{n}=\frac{k_{m} \psi_{n}^{\prime}\left(k_{m} a\right) \psi_{n}\left(k_{p} a\right)-\psi_{n}\left(k_{m} a\right) \psi_{n}^{\prime}\left(k_{p} a\right)}{k_{m} \chi_{n}\left(k_{m} a\right) \psi_{n}^{\prime}\left(k_{p} a\right)-\chi_{n}^{\prime}\left(k_{m} a\right) \psi_{n}\left(k_{p} a\right)} .
$$

The primes in Eqs. (A3) denote a differentiation. The coefficients $a_{n}$ and $b_{n}$ in Eqs. (스) have the following form: particle center locations $\mathbf{r}_{1}$ with $\mathbf{r}_{q}$. Equation (ㅁ) can be modified in a manner similar to Eq. (B1) to give the following objective function $\Lambda\left(k_{\mathrm{eff}}^{2}\right)$ :

$$
\Lambda\left(k_{\mathrm{eff}}^{2}\right)=k_{\mathrm{eff}}^{2}-k_{m}^{2}-\sum_{q=1}^{N}\left[\phi_{q}\left(k_{q}^{2}-k_{m}^{2}\right) \Omega_{q}\left(k_{\mathrm{eff}}^{2}\right)\right],
$$

where the constants $\Omega_{q}$ need to be evaluated. The conditionally averaged field in the integral of Eq. (4) should be evaluated for each $q$ th type of particle. For this purpose, $\left\langle\mathbf{E}(\mathbf{r}) \mid \mathbf{r}_{q}\right\rangle$ can be evaluated by solving Eq. (7) simultaneously for all $q$ spheres. The effective-medium model of Fig. 1 will now have $q+1$ layers for each $q$ th particle. The layers arise from the terms in the summation in Eqs. (B1) and (B2).

A bidisperse system is considered here to demonstrate the above-mentioned methodology. In this case, a first type particle is surrounded by a medium with permittivity $\varepsilon_{m}$ up to a radius $R_{11}$ followed by an effective medium with permittivity $\varepsilon_{\text {eff }, 1}=\varepsilon_{m}+\phi_{1}\left(\varepsilon_{1}-\varepsilon_{m}\right) \Omega_{1}$ up to a radius $R_{12}$ followed by the gross effective medium with permittivity $\varepsilon_{\text {eff }}=\varepsilon_{m}+$ $\phi_{1}\left(\varepsilon_{1}-\varepsilon_{m}\right) \Omega_{1}+\phi_{2}\left(\varepsilon_{2}-\varepsilon_{m}\right) \Omega_{2}$. For a second type particle, the layered structure has the radii and permittivity values of $\varepsilon_{m}, R_{21}, \varepsilon_{\text {eff }, 2}, R_{22}$, and $\varepsilon_{\text {eff }}$. The nondimensional radii can be defined as $\kappa_{q r} \equiv R_{q r} / a_{q}$ for the $r$ th layer around the $q$ th particle $(q=1,2)$. The radii are related to the static structure factors $S_{q r}(\mathbf{0})$ through an expression similar to Eqs. (9) and (10) given by [39]

$$
a_{n}=\frac{\psi_{n}\left(k_{\mathrm{eff}} R\right)\left[\psi_{n}^{\prime}\left(k_{m} R\right)-A_{n} \chi_{n}^{\prime}\left(k_{m} R\right)\right]-k_{m} \psi_{n}^{\prime}\left(k_{\mathrm{eff}} R\right)\left[\psi_{n}\left(k_{m} R\right)-A_{n} \chi_{n}\left(k_{m} R\right)\right]}{\zeta_{n}\left(k_{\mathrm{eff}} R\right)\left[\psi_{n}^{\prime}\left(k_{m} R\right)-A_{n} \chi_{n}^{\prime}\left(k_{m} R\right)\right]-k_{m} \zeta_{n}^{\prime}\left(k_{\mathrm{eff}} R\right)\left[\psi_{n}\left(k_{m} R\right)-A_{n} \chi_{n}\left(k_{m} R\right)\right]}
$$

and

$$
b_{n}=\frac{k_{m} \psi_{n}\left(k_{\mathrm{eff}} R\right)\left[\psi_{n}^{\prime}\left(k_{m} R\right)-B_{n} \chi_{n}^{\prime}\left(k_{m} R\right)\right]-\psi_{n}^{\prime}\left(k_{\mathrm{eff}} R\right)\left[\psi_{n}\left(k_{m} R\right)-B_{n} \chi_{n}\left(k_{m} R\right)\right]}{k_{m} \zeta_{n}\left(k_{\mathrm{eff}} R\right)\left[\psi_{n}^{\prime}\left(k_{m} R\right)-B_{n} \chi_{n}^{\prime}\left(k_{m} R\right)\right]-k_{m} \zeta_{n}^{\prime}\left(k_{\mathrm{eff}} R\right)\left[\psi_{n}\left(k_{m} R\right)-B_{n} \chi_{n}\left(k_{m} R\right)\right]} .
$$

\section{APPENDIX B: EXTENSIONS TO POLYDISPERSE AND/OR MULTIPLE SPECIES COMPOSITES}

Let the composite be made of $N$ different types of spherical particles. Particle type $q$ has a radius $\alpha_{q}$ and a permittivity $\varepsilon_{q}$ and occupies a volume fraction $\phi_{q}$. Note that $\sum \phi_{q}=1-\phi_{m}$, where $\phi_{m}$ is the volume fraction of the matrix. Further, let indicator functions $g_{q}(\mathbf{r})$ indicate the locations of the $q$ th type of particles. The effective permittivity of such a composite can be expressed as

$$
\varepsilon_{\mathrm{eff}}=\varepsilon_{m}+\sum_{q=1}^{N}\left[\left(\varepsilon_{q}-\varepsilon_{m}\right)\left\langle g_{q} \mathbf{E}\right\rangle\right]
$$

The averaged field inside a $q$ th type particle, $\left\langle g_{q} \mathbf{E}\right\rangle(\mathbf{r})$, can be evaluated using Eq. (4) by replacing the subscript $p$ with $q$ and

$$
\kappa_{q r}=\left(\frac{\delta_{q r}-S_{q r}(\mathbf{0})}{\phi_{q}}\right)^{\frac{1}{3}}
$$

In Eq. (B3), $\delta_{q r}$ is the Kronecker delta. The structure factors are defined in the following manner:

$$
S_{q r}=\int\left[P\left(\mathbf{r}, a_{q} \mid \mathbf{0}, a_{r}\right)-P\left(\mathbf{r}, a_{q}\right)\right] \mathrm{d} V_{\mathbf{r}}
$$

Here, $P\left(\mathbf{r}, a_{q} \mid \mathbf{0}, a_{r}\right)$ is the probability density of finding a $q$ th type particle at $\mathbf{r}$ given an $r$ th type of particle at the origin 0. However, evaluation of $S_{q r}(\mathbf{0})$ is rather complicated even for a bidisperse system as discussed in [39]. We will note that for hard-sphere random systems, in the limit as $\phi_{1}+\phi_{2} \rightarrow 0$, $S_{q r}=\delta_{q r}-\phi_{q}\left(a_{q}+a_{r}\right)^{3} / a_{q}^{3}$. 


\section{ACKNOWLEDGMENTS}

R. S. and S. W. would like to thank National Science Foundation for financial support through grants CMMI 0855949, administered as a subcontract by University of Tennessee, Knoxville to Syracuse University, and CBET 1049454.

\section{REFERENCES}

1. J. Biener, G. W. Nyce, A. M. Hodge, M. M. Biener, A. V. Hamza, and S. A. Maier, "Nanoporous plasmonic metamaterials," Adv. Mater. 20, 1211-1217 (2008).

2. V. E. Ferry, J. N. Munday, and H. A. Atwater, "Design considerations for plasmonic photovoltaics," Adv. Mater. 22, 4794-4808 (2010).

3. S. A. Maier, M. L. Brongersma, P. G. Kik, S. Meltzer, A. A. G. Requicha, and H. A. Atwater, "Plasmonics - a route to nanoscale optical devices," Adv. Mater. 13, 1501-1505 (2001).

4. W. A. Murray and W. L. Barnes, "Plasmonic materials," Adv. Mater. 19, 3771-3782 (2007).

5. J. Yao, A. P. Le, S. K. Gray, J. S. Moore, J. A. Rogers, and R. G. Nuzzo, "Functional nanostructured plasmonic materials," Adv. Mater. 22, 1102-1110 (2010).

6. P. R. West, S. Ishii, G. V. Naik, N. K. Emani, V. M. Shalaev, and A. Boltasseva, "Searching for better plasmonic materials," Laser Photon. Rev. 4, 795-808 (2010)

7. T. Cong, S. N. Wani, P. A. Paynter, and R. Sureshkumar, "Structure and optical properties of self-assembled multicomponent plasmonic nanogels,” Appl. Phys. Lett. 99, 043112 (2011).

8. J. Trice, D. Thomas, C. Favazza, R. Sureshkumar, and R. Kalyanaraman, "Pulsed-laser-induced dewetting in nanoscopic metal films: theory and experiments," Phys. Rev. B 75, 235439 (2007)

9. T. Li, J. Moon, A. A. Morrone, J. J. Mecholsky, D. R. Talham, and J. H. Adair, "Preparation of $\mathrm{Ag} / \mathrm{SiO} 2$ nanosize composites by a reverse micelle and sol-gel technique," Langmuir 15, 4328-4334 (1999).

10. D. D. Smith, L. A. Snow, L. Sibille, and E. Ignont, "Tunable optical properties of metal nanoparticle sol-gel composites," J. Non-Cryst. Solids 285, 256-263 (2001).

11. L. M. Liz-Marzán, "Tailoring surface plasmons through the morphology and assembly of metal nanoparticles," Langmuir 22, 32-41 (2006).

12. A. Biswas, O. C. Aktas, U. Schurmann, U. Saeed, V. Zaporojtchenko, F. Faupel, and T. Strunskus, "Tunable multiple plasmon resonance wavelengths response from multicomponent polymer-metal nanocomposite systems," Appl. Phys. Lett. 84, 2655-2657 (2004).

13. Z. Liu, H. Wang, H. Li, and X. Wang, "Red shift of plasmon resonance frequency due to the interacting Ag nanoparticles embedded in single crystal $\mathrm{Sio}_{2}$ by implantation," Appl. Phys. Lett. 72, 1823-1825 (1998).

14. S. Pillai, K. R. Catchpole, T. Trupke, and M. A. Green, "Surface plasmon enhanced silicon solar cells," J. Appl. Phys. 101, 093105-093108 (2007).

15. H. A. Atwater and A. Polman, "Plasmonics for improved photovoltaic devices," Nat. Mater. 9, 205-213 (2010).

16. S. Torkamani, S. N. Wani, Y. J. Tang, and R. Sureshkumar, "Plasmon-enhanced microalgal growth in miniphotobioreactors," Appl. Phys. Lett. 97, 043703-043703 (2010).

17. D. Erickson, D. Sinton, and D. Psaltis, "Optofluidics for energy applications," Nat. Photon. 5, 583-590 (2011)

18. O. Popov, A. Zilbershtein, and D. Davidov, "Random lasing from dye-gold nanoparticles in polymer films: enhanced gain at the surface-plasmon-resonance wavelength," Appl. Phys. Lett. 89,191116 (2006).

19. T. Okamoto, I. Yamaguchi, and T. Kobayashi, "Local plasmon sensor with gold colloid monolayers deposited upon glass substrates," Opt. Lett. 25, 372-374 (2000).

20. A. Dawson and P. V. Kamat, "Semiconductor-metal nanocomposites. Photoinduced fusion and photocatalysis of gold-capped TiO2 (TiO2/Gold) nanoparticles," J. Phys. Chem. B 105, 960-966 (2001).

21. C. F. Bohren and D. R. Huffman, Absorption and Scattering of Light by Small Particles (Wiley, 1998).
22. S. A. Maier, Plasmonics (Springer Science+Business Media, 2007).

23. E. Ozbay, "Plasmonics: merging photonics and electronics at nanoscale dimensions," Science 311, 189-193 (2006).

24. C. Oubre and P. Nordlander, "Optical properties of metallodielectric nanostructures calculated using the finite difference time domain method," J. Phys. Chem. B 108, 17740-17747 (2004).

25. A. Taflove and S. C. Hagness, Computational Electrodynamics: The Finite-Difference Time-Domain Method (Artech House, 2005).

26. F. Kaminski, V. Sandoghdar, and M. Agio, "Finite-difference time-domain modeling of decay rates in the near field of metal nanostructures," J. Comp. Theor. Nanosci. 4, 635-643 (2007)

27. T. C. Choy, Effective Medium Theory (Oxford University, 1999).

28. J. C. M. Garnett, "Colours in metal glasses and in metallic films," Phil. Trans. R. Soc. A 203, 385-420 (1904).

29. P. Mallet, C. A. Guérin, and A. Sentenac, "Maxwell-Garnett mixing rule in the presence of multiple scattering: derivation and accuracy," Phys. Rev. B 72, 014205 (2005).

30. R. Ruppin, "Evaluation of extended Maxwell-Garnett theories," Opt. Commun. 182, 273-279 (2000).

31. V. Yannopapas, "Effective-medium description of disordered photonic alloys," J. Opt. Soc. Am. B 23, 1414-1419 (2006).

32. D. M. Wood and N. W. Ashcroft, "Effective medium theory of the optical properties of small particle composites," Philos. Mag. 35, 269-280 (1977).

33. P. D. M. Spelt, M. A. Norato, A. S. Sangani, M. S. Greenwood, and L. L. Tavlarides, "Attenuation of sound in concentrated suspensions: theory and experiments," J. Fluid Mech. 430, 51-86 (2001).

34. G. Mo and A. S. Sangani, "A method for computing Stokes flow interactions among spherical objects and its application to suspensions of drops and porous particles," Phys. Fluids 6 1637-1652 (1994).

35. T. L. Dodd, D. A. Hammer, A. S. Sangani, and D. L. Koch, "Numerical simulations of the effect of hydrodynamic interactions on diffusivities of integral membrane proteins," J. Fluid Mech. 293, 147-180 (1995).

36. A. S. Sangani and C. Yao, "Bulk thermal conductivity of composites with spherical inclusions," J. Appl. Phys. 63, 1334-1341 (1988).

37. A. S. Sangani, "A pairwise interaction theory for determining the linear acoustic properties of dilute bubbly liquids," J. Fluid Mech. 232, 221-284 (1991)

38. A. S. Sangani and W. Lu, "Elastic coefficients of composites containing spherical inclusions in a periodic array," J. Mech. Phys. Solids 35, 1-21 (1987).

39. S. Koo and A. S. Sangani, "Effective-medium theories for predicting hydrodynamic transport properties of bidisperse suspensions," Phys. Fluids 14, 3522-3533 (2002).

40. N. F. Carnahan and K. E. Starling, "Equation of state for nonattracting rigid spheres," J. Chem. Phys. 51, 635-636 (1969).

41. S. Chandrasekhar, Hydrodynamic and Hydromagnetic Stability (Clarendon Press, 1961).

42. R. F. Harrington, Time-Harmonic Electromagnetic Fields (IEEE, 2001).

43. R. L. Hightower and C. B. Richardson, "Resonant Mie scattering from a layered sphere," Appl. Opt. 27, 4850-4855 (1988).

44. M. Abramowitz and I. A. Stegun, Handbook of Mathematical Functions: With Formulas, Graphs, and Mathematical tables (Dover, 1970).

45. D. Zwillinger, Handbook of Integration (Jones and Bartlett, 1992).

46. C. T. Kelley, Iterative Methods for Linear and Nonlinear Equations (SIAM, 1995).

47. N-k database, http://www.sopra-sa.com

48. A. H. Sihvola, Electromagnetic Mixing Formulas and Applications (Institution of Electrical Engineers, 1999).

49. H. Garcia, J. Trice, R. Kalyanaraman, and R. Sureshkumar, "Selfconsistent determination of plasmonic resonances in ternary nanocomposites," Phys. Rev. B 75, 045439 (2007).

50. N. W. Ashcroft and N. D. Mermin, Solid State Physics (Holt, Rinehart, and Winston, 1976). 
51. M. G. Blaber, M. D. Arnold, and M. J. Ford, "Search for the ideal plasmonic nanoshell: the effects of surface scattering and alternatives to gold and silver," J. Phys. Chem. C 113, 3041-3045 (2009).

52. K. L. Kelly, E. Coronado, L. L. Zhao, and G. C. Schatz, "The optical properties of metal nanoparticles: the influence of size, shape, and dielectric environment," J. Phys. Chem. B 107, 668-677 (2003).

53. See Appendix A.

54. E. D. Palik, Hañdbook of Optical Constants of Solids (Academic Press, 1985).

55. U. Fano, "Effects of configuration interaction on intensities and phase shifts," Phys. Rev. 124, 1866 (1961).
56. N. J. Halas, S. Lal, W. S. Chang, S. Link, and P. Nordlander, "Plasmons in strongly coupled metallic nanostructures," Chem. Rev. 111, 3913-3961 (2011).

57. B. Luk'yanchuk, N. I. Zheludev, S. A. Maier, N. J. Halas, P. Nordlander, H. Giessen, and C. T. Chong, "The Fano resonance in plasmonic nanostructures and metamaterials," Nat. Mater. 9, $707-715$ (2010).

58. A. E. Miroshnichenko, S. Flach, and Y. S. Kivshar, "Fano resonances in nanoscale structures," Rev. Mod. Phys. 82, 2257-2298 (2010).

59. H. Garcia, R. Kalyanaraman, and R. Sureshkumar, "Nonlinear optical properties of multi-metal nanocomposites in a glass matrix,” J. Phys. B 42, 175401 (2009). 